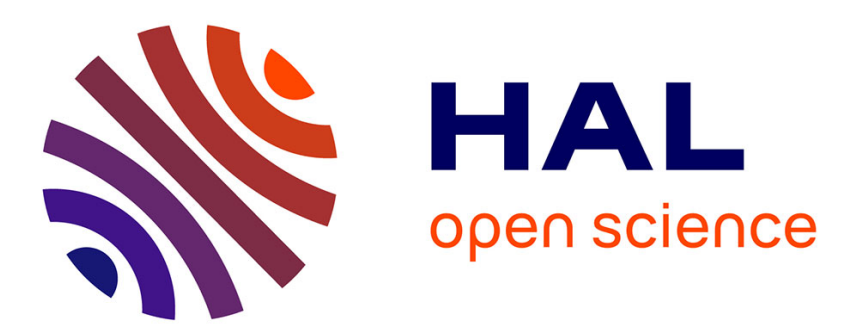

\title{
Effect of solid thermal conductivity and particle-particle contact on effective thermodiffusion coefficient in porous media
}

Hossein Davarzani, Manuel Marcoux, Michel Quintard

\section{- To cite this version:}

Hossein Davarzani, Manuel Marcoux, Michel Quintard. Effect of solid thermal conductivity and particle-particle contact on effective thermodiffusion coefficient in porous media. International Journal of Thermal Sciences, 2011, vol. 50, pp. 2328-2339. 10.1016/j.ijthermalsci.2011.06.016 hal-00918227

\section{HAL Id: hal-00918227 \\ https://hal.science/hal-00918227}

Submitted on 13 Dec 2013

HAL is a multi-disciplinary open access archive for the deposit and dissemination of scientific research documents, whether they are published or not. The documents may come from teaching and research institutions in France or abroad, or from public or private research centers.
L'archive ouverte pluridisciplinaire HAL, est destinée au dépôt et à la diffusion de documents scientifiques de niveau recherche, publiés ou non, émanant des établissements d'enseignement et de recherche français ou étrangers, des laboratoires publics ou privés. 


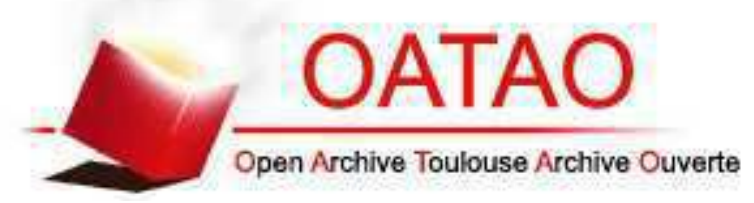

\section{Open Archive TOULOUSE Archive Ouverte (OATAO)}

OATAO is an open access repository that collects the work of Toulouse researchers and makes it freely available over the web where possible.

This is an author-deposited version published in : http://oatao.univ-toulouse.fr/ Eprints ID : 10476

To link to this article : DOI:10.1016/j.ijthermalsci.2011.06.016 URL : http://dx.doi.org/10.1016/j.ijthermalsci.2011.06.016

To cite this version : Davarzani, Hossein and Marcoux, Manuel and Quintard, Michel. Effect of solid thermal conductivity and particle-particle contact on effective thermodiffusion coefficient in porous media. (2011) International Journal of Thermal Sciences, vol. 50 . pp. 2328-2339. ISSN 1290-0729

Any correspondance concerning this service should be sent to the repository administrator: staff-oatao@ listes-diff.inp-toulouse.fr 


\title{
Effect of solid thermal conductivity and particle-particle contact on effective thermodiffusion coefficient in porous media
}

\author{
H. Davarzani ${ }^{a, b}$, M. Marcoux ${ }^{a, b, *}$, M. Quintard ${ }^{a, b}$ \\ ${ }^{a}$ Université de Toulouse, INPT, UPS, IMFT (Institut de Mécanique des Fluides de Toulouse), Allée Camille Soula, F-31400 Toulouse, France \\ ${ }^{\mathrm{b}}$ CNRS, IMFT, F-31400 Toulouse, France
}

Keywords:

Thermodiffusion

Particle-particle contact

Soret effect

Porous media

Two-bulb method

Volume averaging technique

\begin{abstract}
A B S T R A C T
Transient mass transfer associated to a thermal gradient through a saturated porous medium is studied experimentally and theoretically to determine the effect of solid thermal conductivity and particle-particle contact on thermodiffusion processes. In this study, the theoretical volume averaging model developed in a previous study has been adopted to determine the effective transport coefficients in the case of particle-particle contact configurations. The theoretical results revealed that the effective thermodiffusion coefficient is independent of the thermal conductivity ratio for pure diffusive cases. In all cases, even if the effective thermal conductivity depends on the particle-particle contact, the effective thermodiffusion coefficient remains independent of the solid phase connectivity. We also found that the porosity can change the impact of dispersion effects on the thermodiffusion coefficients. For large values of the thermal conductivity contrast, dispersion effects are negligible and the effective thermal conductivity coefficients are the same as the ones for the pure diffusion case.

Experimental results obtained for the purely diffusive case, using a special two-bulb apparatus, confirm the theoretical results. These results also show that, for non-consolidated porous media made of spheres, the thermal conductivity ratio has no significant influence on the thermodiffusion process for pure diffusion. Finally, the particle-particle contact also does not show a considerable influence on the thermodiffusion process.
\end{abstract}

\section{Introduction}

In many situations involving multi-component fluids mixture, the presence of thermal gradient can be the cause of species migration because of the phenomenon known as Soret effect or thermodiffusion $[25,41]$. The applications of thermodiffusion in free fluid as well as in porous media become more and more important nowadays in industrial such as isotope separation in liquid and gaseous mixtures [33,34], polymer solutions and colloidal dispersions [45], study of compositional variation in hydrocarbon reservoirs [12], coating of metallic items, solidifying metallic alloys, migration of the DNA molecules [3,23], volcanic lava, transport in the Earth Mantle [19], etc.

Many works have been carried out to determine thermodiffusion coefficients using different approaches. Experimental approaches have been developed for instance using a two-bulb method in the case of a free medium (Ibbs and Chapman, 1921 [18]; Heath et al., 1941 [16]; van Itterbeek et al., 1947 [43]; Mason et al., 1964 [26]; Saxena et al., 1966 [38]; Humphreys and Gray, 1970 [17]; Grew and Wakeham, 1971

\footnotetext{
* Corresponding author. Université de Toulouse, INPT, UPS, IMFT (Institut de Mécanique des Fluides de Toulouse), Allée Camille Soula, F-31400 Toulouse, France Tel.: +33 (0) 534322873 ; fax: + 33 (0) 534322899 .

E-mail address: marcoux@imft.fr (M. Marcoux).
}

[15]; Shashkov et al., 1979 [40] and Zhdanov et al., 1980 [46]). Soret coefficients have also been determined in crude oils under microgravity condition [14,42] or thermogravitational column [29]. Theoretical approaches have also been used like molecular dynamics simulations [13,35] or multi-component numerical models [36].

While the transfer of species related to thermodiffusion in porous media may directly be linked to the physical and thermal properties of the solid structures, various theoretical studies had generally treated this problem considering the whole system as an equivalent continuum (macroscopic approach) $[1,21]$. Failures in the thermogravitational models [11] based on the free fluid equations are a good example of the need to determine the influence of the thermo-physical properties of the pore matrix on the thermodiffusion process in porous media.

Additionally, one of the most important features that has not really been taken into account is the existing difference in thermal conductivity between the solid matrix and the fluid phase.

The evaluation of the influence of the porosity and tortuosity on the thermodiffusion coefficients has been the object of many researches:

- Experimental studies: Platten and Costesèque (2004), using a thermogravitational column [29]; Costesèque et al.(2004), using 


\begin{tabular}{|c|c|c|c|}
\hline \multicolumn{2}{|c|}{ Nomenclature } & $\mathbf{r}$ & Position vector, $\mathrm{m}$ \\
\hline \multirow[b]{2}{*}{$\mathscr{A}_{\beta \sigma}$} & & $\boldsymbol{t}$ & \\
\hline & $\begin{array}{l}\text { Area of the } \beta-\sigma \text { interface contained within the } \\
\text { macroscopic region, } \mathrm{m}^{2}\end{array}$ & $\begin{array}{l}T_{\beta} \\
\left\langle T_{\beta}\right\rangle^{\beta}\end{array}$ & $\begin{array}{l}\text { Temperature of the } \beta \text {-phase, } \mathrm{K} \\
\text { Intrinsic average temperature of the } \beta \text {-phase, } \mathrm{K}\end{array}$ \\
\hline$A_{\beta \sigma}$ & $\begin{array}{l}\text { Area of the } \beta-\sigma \text { interface within the averaging } \\
\text { volume, } \mathrm{m}^{2}\end{array}$ & $\begin{array}{l}\dot{\tilde{T}}_{\beta} \\
T_{H}, T_{C}\end{array}$ & $\begin{array}{l}\text { Spatial deviation temperature, } K \\
\text { Hot and cold temperature }\end{array}$ \\
\hline a/d & Particle-particle contact fraction & $p_{\beta}$ & Pressure of the $\beta$-phase, $\mathrm{Pa}$ \\
\hline $\mathbf{b}_{C \beta}$ & Mapping vector field for $\tilde{c}_{\beta}, \mathrm{m}$ & $\mathrm{Pe}$ & Unit cell Péclet number \\
\hline $\mathbf{b}_{S \beta}$ & Mapping vector field for $c_{\beta}, \mathrm{m}$ & $\mathbf{v}_{\beta}$ & Mass average velocity of the $\beta$-phase, $\mathrm{m} / \mathrm{s}$ \\
\hline $\mathbf{b}_{T \beta}$ & Mapping vector field for $\tilde{T}_{\beta}, \mathrm{m}$ & $\left\langle\mathbf{v}_{\beta}\right\rangle^{\beta}$ & Intrinsic mass average velocity of the $\beta$-phase, $\mathrm{m} / \mathrm{s}$ \\
\hline$c_{p}$ & Heat capacity, J/kg K & $\tilde{\mathbf{v}}_{\beta}$ & Spatial deviation mass average velocity, $\mathrm{m} / \mathrm{s}$ \\
\hline $\begin{array}{l}c_{\beta} \\
\left\langle c_{\beta}\right\rangle^{\beta}\end{array}$ & $\begin{array}{l}\text { Total mass fraction of the } \beta \text {-phase } \\
\text { Intrinsic average mass fraction of the } \beta \text {-phase }\end{array}$ & $V_{\beta}$ & $\begin{array}{l}\text { Volume of the } \beta \text {-phase contained within the averaging } \\
\text { volume, } \mathrm{m}^{3}\end{array}$ \\
\hline$\hat{c}_{\beta}$ & Spatial deviation mass fraction of the $\beta$-phase & $\mathscr{V}$ & Local averaging volume, $\mathrm{m}^{3}$ \\
\hline$D_{\beta}$ & Binary diffusion coefficient, $\mathrm{m}^{2} / \mathrm{s}$ & $x, y$ & Cartesian coordinates, m \\
\hline $\mathbf{D}_{\beta}^{*}$ & Effective dispersion tensor, $\mathrm{m}^{2} / \mathrm{s}$ & & \\
\hline$\left.\mathrm{D}_{\beta}^{*}\right|_{\chi x}$ & Longitudinal dispersion coefficient, $\mathrm{m}^{2} / \mathrm{s}$ & \multicolumn{2}{|c|}{ Greek symbols } \\
\hline$D_{T \beta}^{p a x}$ & Thermodiffusion coefficient, $\mathrm{m}^{2} / \mathrm{s} \mathrm{K}$ & $\varepsilon_{\beta}$ & Volume fraction of the $\beta$-phase or porosity \\
\hline $\mathbf{D}_{T \beta}^{*}$ & Effective thermodiffusion tensor, $\mathrm{m}^{2} / \mathrm{s} \mathrm{K}$ & $\varepsilon_{\sigma}$ & Volume fraction of the $\sigma$-phase or porosity \\
\hline$\left.\mathrm{D}_{T \beta}^{*}\right|_{\chi x x}$ & Longitudinal thermodiffusion coefficient, $\mathrm{m}^{2} / \mathrm{s} \mathrm{K}$ & $\kappa$ & $k_{\sigma} / k_{\beta}$, conductivity ratio \\
\hline $\mathbf{g}$ & Gravitational acceleration, $\mathrm{m}^{2} / \mathrm{s}$ & $\mu_{\beta}$ & Dynamic viscosity for the $\beta$-phase, Pa s \\
\hline$k_{\beta}$ & Thermal conductivity of the fluid phase, $\mathrm{W} / \mathrm{m} \mathrm{K}$ & $\rho_{\beta}$ & Total mass density in the $\beta$-phase, $\mathrm{kg} / \mathrm{m}^{3}$ \\
\hline$k_{\sigma}$ & Thermal conductivity of the solid phase, $\mathrm{W} / \mathrm{m} \mathrm{K}$ & $\tau$ & Scalar tortuosity factor \\
\hline $\mathbf{k}^{*}$ & Effective thermal conductivity tensor, $\mathrm{W} / \mathrm{m} \mathrm{K}$ & $\varphi$ & Arbitrary function \\
\hline$\left.\mathbf{k}^{*}\right|_{\not X X}$ & Longitudinal thermal dispersion coefficient, W/m K & & \\
\hline$\ell_{U C}$ & Characteristic length scale associated with a unit & \multicolumn{2}{|c|}{ Subscripts, superscripts and other symbols } \\
\hline & cell, m & $\beta$ & Fluid-phase \\
\hline$L$ & Characteristic length for macroscopic quantities, $m$ & $\beta \sigma$ & $\beta-\sigma$ interface \\
\hline$\ell_{\beta}$ & Characteristic length for the $\beta$-phase, $\mathrm{m}$ & $\sigma$ & Solid-phase \\
\hline $\mathbf{n}_{\beta \sigma}$ & Unit normal vector directed from the $\beta$-phase towards & & Effective quantity \\
\hline & the $\sigma$-phase & \langle\rangle & Spatial average \\
\hline$r_{0}$ & Radius of the averaging volume, $\mathrm{m}$ & \langle\rangle$^{\varphi}$ & Intrinsic $\varphi$-phase average \\
\hline
\end{tabular}

free and porous packed thermodiffusion cells [5]; Davarzani et al. (2010), using a two-bulb setup [8],

- Theoretical approaches: A. A. Shapiro and E. H. Stenby (2000), using the theorem of factorization [39]; Lacabanne et al. (2002), using an homogenization technique [24]; Galliero et al. (2006), using molecular dynamics computer simulations [13]; Davarzani et al. (2010), using a volume averaging technique [9].

The common point of these results is that the tortuosity factor acts in the same way on the Fick diffusion coefficient and on the thermodiffusion coefficient. Recently, the theoretical results of the model developed by Davarzani et al. (2010) showed that this concept is correct only in a pure diffusive regime. They used a volume averaging technique to determine the effective diffusion and thermodiffusion coefficients in more complex conditions including convective regimes. The results showed that, when convection is dominant (convective regime), Soret coefficients in a porous medium are very different from the ones in a free fluid. More precisely, the impact of convection on the mass transfer by thermodiffusion is different from mass transfer by dispersion [9]. For this regime, the ratio of the phases conductivity also has a great influence on the effective thermodiffusion coefficients. Therefore, using the same Soret number in porous medium and free fluid may cause significant errors.

It is known theoretically and experimentally that heat conduction at the contact points plays a dominant role in determining the effective heat conduction in porous media [27,28,31,37]. Especially, a theory of heat conduction in two-phase systems considering also the particle-particle contact, has been presented by Nozad et al.
(1985), based on the method of volume averaging for spatially periodic porous media. The results showed that the effective thermal conductivity for non-touching particles is dominated by the thermal conductivity ratio and also by porosity, and is only slightly influenced by the geometrical configuration (see [27]). In an experimental study done by Nozad et al. (1985) [28], the effective thermal conductivity coefficients have been measured for different materials and fluid systems. For example, they used the aluminium-water system to study the influence of non-touching particles using the fact that water tends to react with aluminium to form an oxide layer with a low conductivity. Whereas, an air-aluminium system has been used to model the particle-particle contact. When the fluid phase is continuous, the effective thermal conductivity coefficients become constant for large values of the thermal conductivity ratio. The situation for touching particles or a continuous solid phase is very different. In this case, at large values of the thermal conductivity ratio, there is a linear dependence of the effective coefficients on the thermal conductivity ratio. The comparison presented by Nozad et al. (1985) showed a good agreement between theory and experiments [27]. Sahraoui and Kaviany (1993) repeated the computation of Nozad et al. and found the contact fraction ratio that gives the closest agreement with the available experiment data [37]. However, the influence on thermodiffusion has not been investigated.

In this work, we study the influence of the conductivity ratio and particle-particle contact on the effective thermodiffusion coefficients from theoretical approach and experimental measurements. To achieve this objective, we have developed a model based on the 
equations and closure problems developed by Davarzani et al. (2010) that describe thermodiffusion processes in a homogeneous porous medium by upscaling technique. This model allows characterizing the influence of the porous matrix properties on the thermodiffusion process [9]. In order to understand the effect of particle-particle contact, we solved the closure problems for temperature and concentration on a particular geometry in which the solid phase is continuous through small contact areas.

Then, to validate these theoretical results, new experimental results have been obtained with a special two-bulb apparatus. The thermodiffusion of a helium-carbon dioxide mixture through cylindrical samples filled with porous media made of spheres with different thermal properties (stainless steel, glass and aluminium) has been measured at the atmospheric pressure.

\section{Governing microscopic equations}

We consider in this study a porous medium saturated with a binary mixture fluid and subjected to a thermal gradient. This system is illustrated in Fig. 1, the fluid phase is identified as the $\beta$-phase while the rigid solid phase is represented by the $\sigma$-phase.

The equations of continuity and motion have to be introduced for the fluid phase. We use Stokes equation for the flow motion at the pore-scale, assuming classically that inertia effects can be neglected. The continuity equation, the Stokes equation, and the no-slip boundary condition are then written as

$\nabla \cdot \mathbf{v}_{\beta}=0$, in the $\beta-$ phase

$\mathbf{0}=-\nabla p_{\beta}+\mu_{\beta} \nabla \cdot\left(\nabla \mathbf{v}_{\beta}\right)+\rho_{\beta} \mathbf{g}$, in the $\beta-$ phase

$\mathbf{v}_{\beta}=0$, at $\mathscr{A}_{\beta \sigma}$

In this problem, we also consider the flow of the $\beta$-phase to be steady, incompressible, and laminar.

From the thermodynamics of irreversible processes as originally formulated by Onsager (1931) the diagonal effects that describe heat and mass transfer are Fourier's law which relates heat flow to the temperature gradient and Fick's law which relates mass flow to the concentration gradient. There are also cross effects or coupledprocesses: the Dufour effect quantifies the heat flux caused by the concentration gradient and, conversely, the Soret effect, quantifies the mass flux caused by the temperature gradient.

In this study, we neglect the Dufour effect, which can be justified to be negligible in the case of thermodiffusion process where the concentration gradient is small [30]. Therefore, the pore-scale

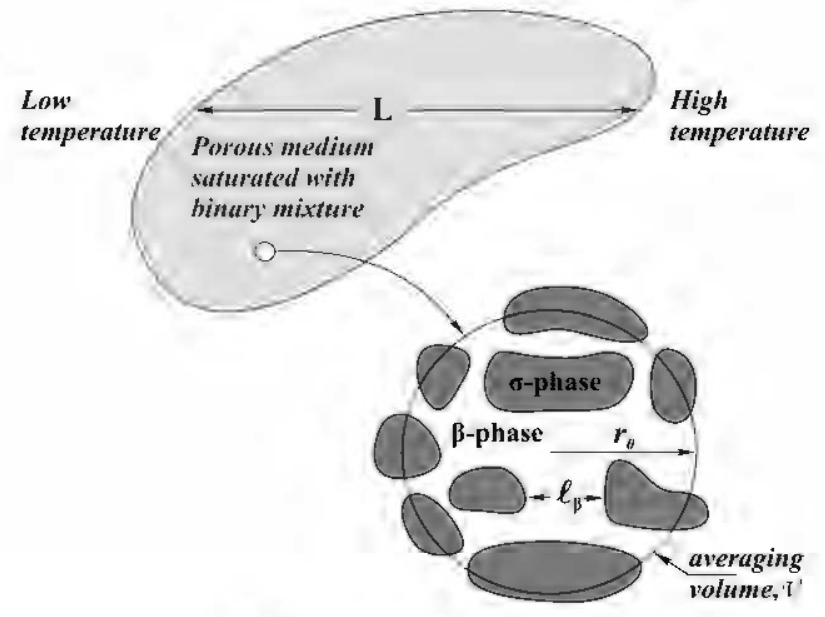

Flg. 1. Problem configuration. transport of energy (conduction and convection) is described by the following equations and boundary conditions for the fluid $(\beta$-phase) and solid ( $\sigma$-phase) phases

$$
\begin{aligned}
& \left(\rho c_{p}\right)_{\beta} \frac{\partial T_{\beta}}{\partial t}+\left(\rho c_{p}\right)_{\beta} \nabla \cdot\left(T_{\beta} \mathbf{v}_{\beta}\right)=\nabla \cdot\left(k_{\beta} \nabla T_{\beta}\right), \text { in the } \beta-\text { phase } \\
& \left(\rho c_{p}\right)_{\sigma} \frac{\partial T_{\sigma}}{\partial t}=\nabla \cdot\left(k_{\sigma} \nabla T_{\sigma}\right), \text { in the } \sigma-\text { phase } \\
& T_{\beta}=T_{\sigma}, \text { at } \mathscr{A}_{\beta \sigma} \\
& \mathbf{n}_{\beta \sigma} \cdot\left(k_{\beta} \nabla T_{\beta}\right)=\mathbf{n}_{\beta \sigma} \cdot\left(k_{\sigma} \nabla T_{\sigma}\right), \text { at } \mathscr{A}_{\beta \sigma}
\end{aligned}
$$

where $\mathscr{A}_{\beta \sigma}$ is the surface of the $\beta-\sigma$ interface contained within the macroscopic region.

The component pore-scale mass transport (diffusion, advection and thermodiffusion) is described by the following mass balance equation in the fluid phase and boundary condition [2]

$$
\begin{aligned}
& \frac{\partial c_{\beta}}{\partial t}+\nabla \cdot\left(c_{\beta} \mathbf{v}_{\beta}\right)=\nabla \cdot\left(D_{\beta} \nabla c_{\beta}+D_{T \beta} \nabla T_{\beta}\right), \text { in the } \beta-\text { phase } \\
& \mathbf{n}_{\beta \sigma} \cdot\left(D_{\beta} \nabla c_{\beta}+D_{T \beta} \nabla T_{\beta}\right)=0 \text {, at } \mathscr{A}_{\beta \sigma}
\end{aligned}
$$

for an impervious boundary, where $c_{\beta}$ is the mass fraction of one component in the $\beta$-phase, $D_{\beta}$ and $D_{T \beta}$ are the molecular isothermal diffusion coefficient and thermodiffusion coefficient, respectively, $\mathbf{n}_{\beta \sigma}$ is the unit normal vector pointing from the fluid to the solid phase. We neglect any accumulation and reaction of solute at the fluid-solid interface as well as the phenomenon of surface diffusion (inert solid).

\section{Volume averaging method and macroscopic equations}

In this section, we review briefly the results obtained in a previous paper, Davarzani et al. (2010).

The direct resolution of microscopic equations on a volume containing a small numbers of pores is usually possible and interesting for reasons of fundamental research (e.g. validation of macroscopic models). However, it is generally impossible to solve these microscopic equations on a large volume. In practice, a macroscopic description representing the effective behaviour of the porous medium for a representative elementary volume (REV) containing many pores is needed. Many techniques have been used to move from the pore-scale to the REV scale [6]. Integration on the REV (called volume averaging technique) of the microscopic conservation equations allows obtaining macroscopic equations which are valid for average variables called macroscopic variables [7,9]. We will use such a technique in this paper and the problems associated with upscaling from the microscopic scale to the macroscopic scale will be treated in the next section. This technique will provide the macroscopic equations and the effective transport properties.

The associated averaging volume, $r$ is shown in Fig. 1 . The development of local volume averaged equations requires that we define two types of averages in terms of the averaging volume [44]. For any quantity $\varphi_{\beta}$ associated with the $\beta$-phase, the superficial average is defined according to

$$
\left\langle\varphi_{\beta}\right\rangle=\frac{1}{\mathscr{V}} \int_{V_{\beta}} \varphi_{\beta} \mathrm{d} V
$$

while the intrinsic average is defined by

$\left\langle\varphi_{\beta}\right\rangle^{\beta}=\frac{1}{V_{\beta}} \int_{V_{\beta}} \varphi_{\beta} d V$ 
Here we have used $V_{\beta}$ to represent the volume of the $\beta$-phase contained within the averaging volume. These two averages are related by

$$
\left\langle\varphi_{\beta}\right\rangle=\varepsilon_{\beta}\left\langle\varphi_{\beta}\right\rangle^{\beta}
$$

in which $\varepsilon_{\beta}$ is the volume fraction of the $\beta$-phase or porosity in the one phase flow case.

The sum of volume fractions of the two phases satisfies

$\varepsilon_{\beta}+\varepsilon_{\sigma}=1$

Following classical ideas [44] we will solve the problems in terms of averaged values and deviations. The pore-scale field deviations in the $\beta$-phase and $\sigma$-phase are respectively defined by

$\varphi_{\beta}=\left\langle\varphi_{\beta}\right\rangle^{\beta}+\tilde{\varphi}_{\beta}$ and $\varphi_{\sigma}=\left\langle\varphi_{\sigma}\right\rangle^{\sigma}+\tilde{\varphi}_{\sigma}$

Classical length-scale constraints (Fig. 1) will be imposed by assuming

$\ell_{\beta}<<r_{0}<<L$

After performing the volume averaging on the original boundary value problem and solving the associated closure problems, the final form of the transport equations contains local averages, rather than micro-scale point values. Thus, the microscopic equations that hold for a point in space are developed into the appropriate macroscopic equations, which hold at a given point for some volume in space of the porous medium. In this study we have used the model of non-isothermal mass transfer with Soret effect developed by Davarzani et al. (2010) and adapted it to a particle-particle contact configuration. Sections 3.1 and 3.2 contain the model equations and closure problems for temperature and concentration respectively. The reader can read reference [9] for more details, in particular the derivation of the upscaled equations.

\subsection{Transport equation for the averaged temperature}

When the principle of local-scale heat equilibrium is valid, the one-equation equilibrium model which consists of a single transport equation for a mixture temperature, $\langle T\rangle$, such as $\langle T\rangle \approx\left\langle T_{\beta}\right\rangle^{\beta} \approx\left\langle T_{\sigma}\right\rangle^{\sigma}$ is written $[31,32]$

$$
\begin{aligned}
& \left(\varepsilon_{\beta}\left(\rho c_{p}\right)_{\beta}+\varepsilon_{\sigma}\left(\rho c_{p}\right)_{\sigma}\right) \frac{\partial}{\partial t}\langle T\rangle+\left(\rho c_{p}\right)_{\beta} \nabla \cdot\left(\varepsilon_{\beta}\left\langle\mathbf{v}_{\beta}\right\rangle^{\beta}\langle T\rangle\right) \\
& =\nabla \cdot\left(\mathbf{k}^{*} \cdot \nabla\langle T\rangle\right)
\end{aligned}
$$

where $\mathbf{k}^{*}$ is the effective thermal conductivity given by

$\mathbf{k}^{*}=\left(\varepsilon_{\beta} k_{\beta}+\varepsilon_{\sigma} k_{\sigma}\right) \mathbf{I}+\frac{\left(k_{\beta}-k_{\sigma}\right)}{\mathrm{V}} \int_{A_{\beta \sigma}} \mathbf{n}_{\beta \sigma} \mathbf{b}_{T \beta} d A-\left(\rho c_{p}\right)_{\beta}\left\langle\tilde{\mathbf{v}}_{\beta} \mathbf{b}_{T \beta}\right\rangle$

This result comes from linear relations between the spatial deviations and average temperature of the type [4]

$\tilde{T}_{\beta}=\mathbf{b}_{T \beta} \cdot \nabla\langle T\rangle$

$\tilde{T}_{\sigma}=\mathbf{b}_{T \sigma} \cdot \nabla\langle T\rangle$

in which $\mathbf{b}_{T \beta}$ and $\mathbf{b}_{T \sigma}$ are referred to as the closure mapping variables for the solid and liquid phases, respectively. These mapping vectors are functions of position only, since the time dependence of
$\tilde{T}_{\beta}$ and $\tilde{T}_{\sigma}$ comes only from the time dependence of the average temperature appearing in the equations and boundary conditions for the spatial deviations.

The closure equations for thermal conductivity are listed below, together with their boundary conditions at the fluid-solid interfaces $[9,31,44]$.

Problem I:

$\left(\rho c_{p}\right)_{\beta} \mathbf{v}_{\beta} \cdot \nabla \mathbf{b}_{T \beta}+\left(\rho c_{p}\right)_{\beta} \tilde{\mathbf{v}}_{\beta}=k_{\beta} \nabla^{2} \mathbf{b}_{T \beta}$

$0=k_{\sigma} \nabla^{2} \mathbf{b}_{T \sigma}$

$\mathbf{b}_{T \beta}=\mathbf{b}_{T \sigma}$, at $A_{\beta \sigma}$

$-\mathbf{n}_{\beta \sigma} \cdot k_{\beta} \nabla \mathbf{b}_{T \beta}=-\mathbf{n}_{\beta \sigma} \cdot k_{\sigma} \nabla \mathbf{b}_{T \sigma}+\mathbf{n}_{\beta \sigma} \cdot\left(k_{\beta}-k_{\sigma}\right)$, at $A_{\beta \sigma}$

$\mathbf{b}_{T \beta}\left(\mathbf{r}+\ell_{i}\right)=\mathbf{b}_{T \beta}(\mathbf{r}) \& \mathbf{b}_{T \sigma}\left(\mathbf{r}+\ell_{i}\right)=\mathbf{b}_{T \sigma}(\mathbf{r}), i=1,2,3$

$\left\langle\mathbf{b}_{T}\right\rangle=\varepsilon_{\beta}\left\langle\mathbf{b}_{T \beta}\right\rangle^{\beta}+\varepsilon_{\sigma}\left\langle\mathbf{b}_{T \sigma}\right\rangle^{\sigma}=0$

We obtained that the boundary condition presented in Equation (23) can be also replaced by

$\mathbf{n}_{\beta \sigma} \cdot k_{\beta} \nabla \mathbf{b}_{T \beta}=\mathbf{0}$, at $A_{\beta \sigma}$

$\mathbf{n}_{\beta \sigma} \cdot k_{\sigma} \nabla \mathbf{b}_{T \sigma}=\mathbf{n}_{\beta \sigma} \cdot\left(k_{\beta}-k_{\sigma}\right)$, at $A_{\beta \sigma}$

or

$\mathbf{n}_{\beta \sigma} \cdot k_{\beta} \nabla \mathbf{b}_{T \beta}=-\mathbf{n}_{\beta \sigma} \cdot k_{\beta}$, at $A_{\beta \sigma}$

$\mathbf{n}_{\beta \sigma} \cdot k_{\sigma} \nabla \mathbf{b}_{T \sigma}=-\mathbf{n}_{\beta \sigma} \cdot k_{\sigma}$, at $A_{\beta \sigma}$

\subsection{Transport equation for averaged concentration}

The closed averaged form of the mass transfer equation with Soret effect was obtained as [9]

$\frac{\partial\left\langle\varepsilon_{\beta} c_{\beta}\right\rangle^{\beta}}{\partial t}+\nabla \cdot\left(\varepsilon_{\beta}\left\langle\mathbf{v}_{\beta}\right\rangle^{\beta}\left\langle c_{\beta}\right\rangle^{\beta}\right)=\nabla \cdot\left(\varepsilon_{\beta} \mathbf{D}_{\beta}^{*} \cdot \nabla\left\langle c_{\beta}\right\rangle^{\beta}+\varepsilon_{\beta} \mathbf{D}_{T \beta}^{*} \cdot \nabla\langle T\rangle\right)$

where the total dispersion and total thermodiffusion tensors are defined by

$$
\begin{aligned}
\mathbf{D}_{\beta}^{*}= & D_{\beta}\left(\mathbf{I}+\frac{1}{V_{\beta}} \int_{A_{\beta \sigma}} \mathbf{n}_{\beta \sigma} \mathbf{b}_{C \beta} d A\right)-\left\langle\tilde{\mathbf{v}}_{\beta} \mathbf{b}_{C \beta}\right\rangle^{\beta} \\
\mathbf{D}_{T \beta}^{*}= & D_{\beta}\left(\frac{1}{V_{\beta}} \int_{A_{\beta \sigma}} \mathbf{n}_{\beta \sigma} \mathbf{b}_{S \beta} d A\right)+D_{T \beta}\left(\mathbf{I}+\frac{1}{V_{\beta}} \int_{A_{\beta \sigma}} \mathbf{n}_{\beta \sigma} \mathbf{b}_{T \beta} d A\right) \\
& -\left\langle\tilde{\mathbf{v}}_{\beta} \mathbf{b}_{S \beta}\right\rangle^{\beta}
\end{aligned}
$$

This result has been obtained by adopting the following closure relationship

$\tilde{c}_{\beta}=\mathbf{b}_{C \beta} \cdot \nabla\left\langle c_{\beta}\right\rangle^{\beta}+\mathbf{b}_{S \beta} \cdot \nabla\langle T\rangle$ 
in which $\mathbf{b}_{C \beta}$ and $\mathbf{b}_{S \beta}$ are referred to as the closure variables which are specified by the following boundary value problems (see [9] for model validity and details).

Problem IIa:

$\mathbf{v}_{\beta} \cdot \nabla \mathbf{b}_{C \beta}+\tilde{\mathbf{v}}_{\beta}=D_{\beta} \nabla^{2} \mathbf{b}_{C \beta}$

$-\mathbf{n}_{\beta \sigma} \cdot D_{\beta} \nabla \mathbf{b}_{C \beta}=\mathbf{n}_{\beta \sigma} D_{\beta}$, at $A_{\beta \sigma}$

$\mathbf{b}_{C \beta}\left(\mathbf{r}+\ell_{i}\right)=\mathbf{b}_{C \beta}(\mathbf{r}), i=1,2,3$

$\left\langle\mathbf{b}_{C \beta}\right\rangle^{\beta}=\mathbf{0}$

Problem IIb:

$\mathbf{v}_{\beta} \cdot \nabla \mathbf{b}_{S \beta}=D_{\beta} \nabla^{2} \mathbf{b}_{S \beta}+D_{T \beta} \nabla^{2} \mathbf{b}_{T \beta}$

$-\mathbf{n}_{\beta \sigma} \cdot\left(D_{\beta} \nabla \mathbf{b}_{S \beta}+D_{T \beta} \nabla \mathbf{b}_{T \beta}\right)=\mathbf{n}_{\beta \sigma} \cdot D_{T \beta}$, at $A_{\beta \sigma}$

$\mathbf{b}_{S \beta}\left(\mathbf{r}+\ell_{i}\right)=\mathbf{b}_{S \beta}(\mathbf{r}), i=1,2,3$

$\left\langle\mathbf{b}_{\mathrm{s} \beta}\right\rangle^{\beta}=\mathbf{0}$

Problem Ilb couples the heat transfer closure problem to the concentration problem.

We will use later the closure problems to determine the effective parameters for given pore-scale geometries.

We present in the next section, the experimental setup used to measure the effective transport properties.

\section{Experimental setup}

In a previous study, we have designed and built a two-bulb apparatus to obtain directly the diffusion and thermodiffusion coefficients of gases in free fluids as well as in porous media, as described in detail in reference [8].

This setup is a closed system, convenient for the case of pure diffusion only. The two-bulb apparatus used to determine the thermodiffusion coefficients is illustrated in Fig. 2.

The reservoirs which are all-glass bulbs with equal and constant volume of $1 \mathrm{~L}$ are joined by an insulated rigid glass tube. A temperature gradient is setup in the tube by bringing the bulbs to

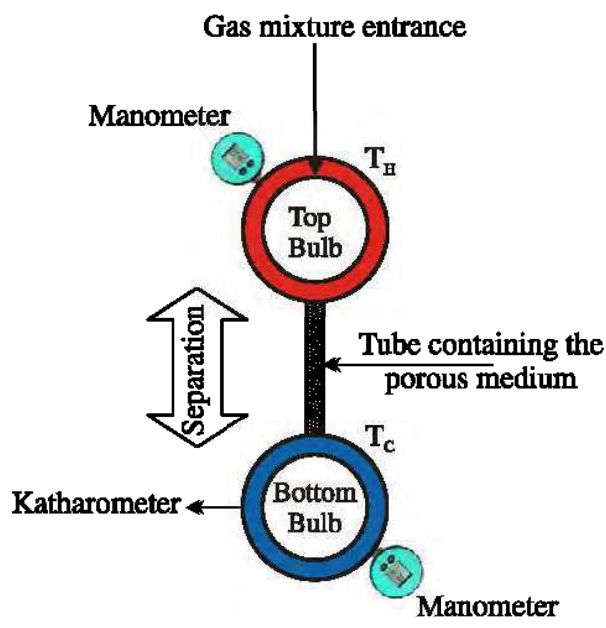

Fig 2. Experimental thermodiffusion two-bulb setup.

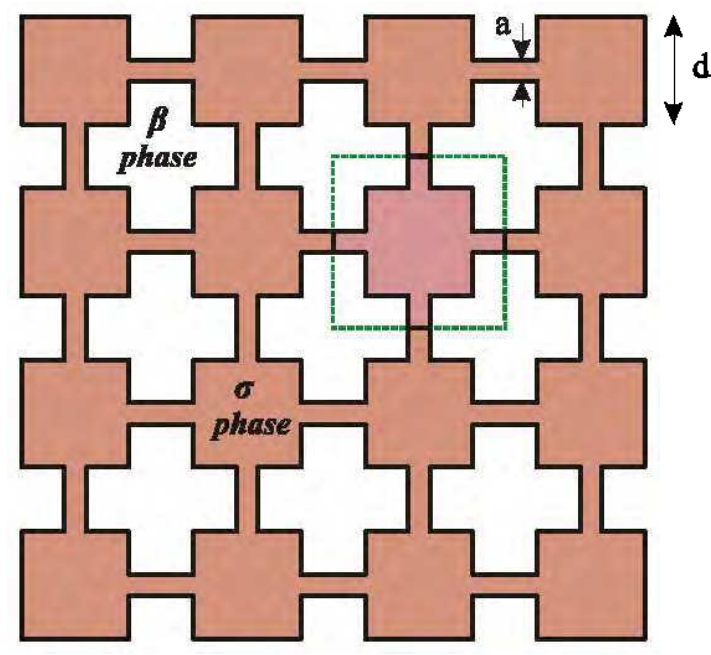

Fig. 3. Spatially periodic model for solid-solid contact.

different temperatures, uniform over each bulb. To avoid convection, the apparatus was mounted vertically, with the hotter bulb uppermost.

Pressure and temperature measurements are made with two manometers and thermometers. The temperature of each bulb is kept at a constant value by water circulation from a bath temperature controller. The concentration of the binary gas mixtures is determined by analysing the gas mixture composition in each bulb using a katharometer with $\pm 0.5 \%$ repeatability (Daynes, 1933 [10] and Jessop, 1966 [20]). Katharometer can indicate continuously and without sampling the changes in composition as thermodiffusion proceeds. The temperature of the katharometer is controlled using an electrical self-thermostated sensor.

In this study, for all thermodiffusion measurements, the temperature difference between the two bulbs is fixed to $50 \mathrm{~K}$. The gas mixture used is $\mathrm{He}-\mathrm{CO}_{2}$. The volume fraction of each gas in the mixture is equal to $50 \%$.

The experiments have been performed with this special twobulb apparatus, by the measurement of the change in bulbs concentration.

\section{Results}

In this section, we present results for the effective coefficients coming from the theoretical (Section 5.1) and experimental

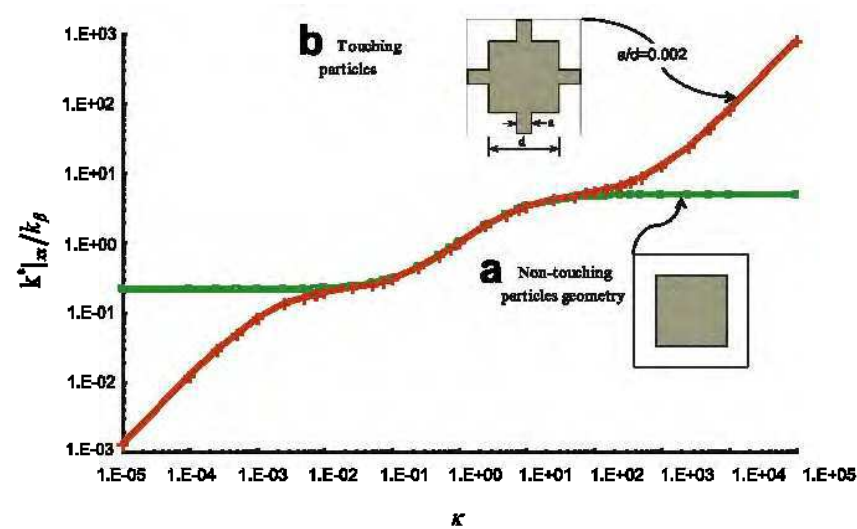

Fig 4. Effective thermal conductivity for (a) non-touching particles, ajd = 0 (b) touching particles, $a / d=0.002$ ( $e_{\beta}=0.36$, for pure diffusion) 


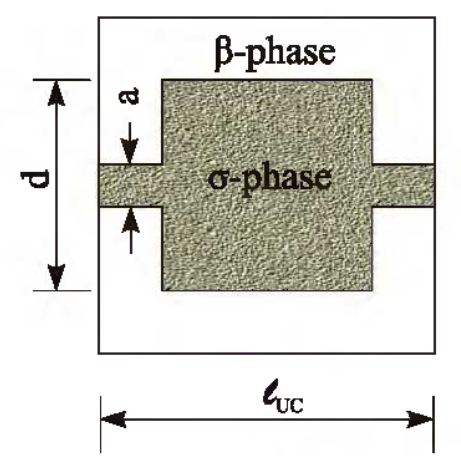

Fig. 5. Spatially periodic unit cell to solve the thermodiffusion closure problem with solid-solid connections $a / d=0.002\left(\varepsilon_{\beta}=0.36\right)$.

(Section 5.2) studies. The numerical simulations of the closure problems have been done using the COMSOL'M Multiphysics finite elements code. The PDEs to be solved are rather classical. Therefore, we do not detail the numerical parameters chosen to achieve numerical convergence and accuracy.
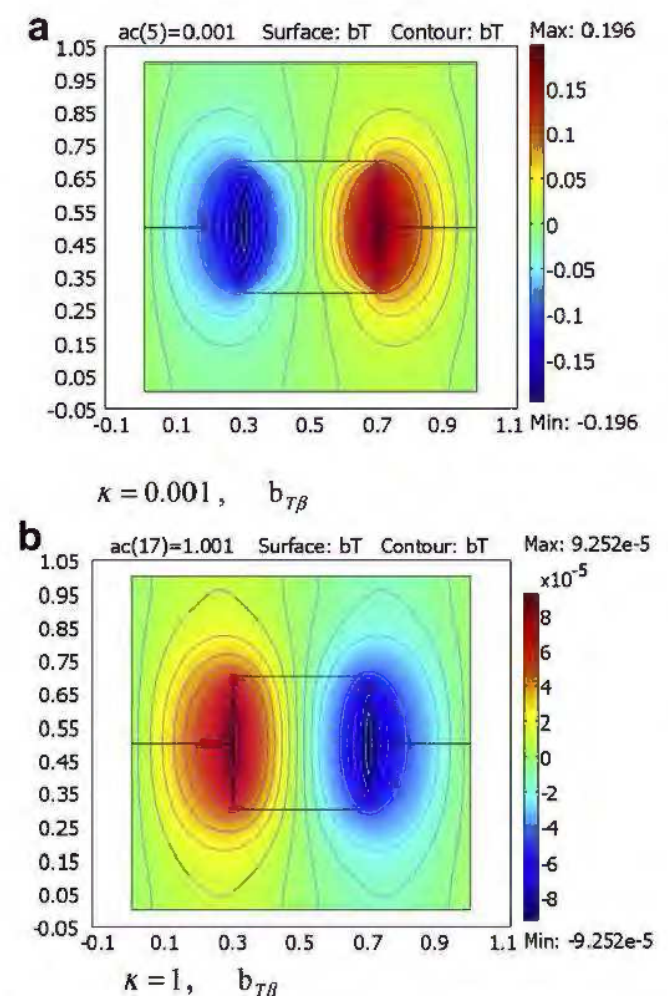

C

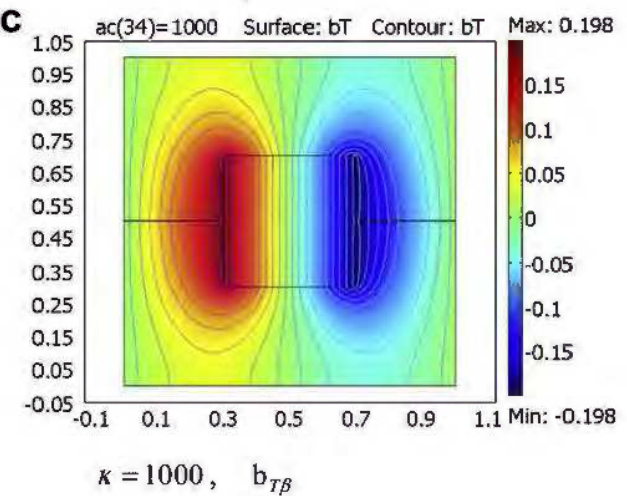

In the theoretical and experimental part, the influence of the thermal conductivity and the particle-particle contact on the kinetic of mass transfer with thermodiffusion has been studied. Then, the experimental results are compared with those obtained using the volume averaging technique in the purely diffusive case.

\subsection{Theoretical results}

The effective coefficients can be obtained by solving numerically the associated closure problem (Problem I for effective thermal conductivity and Problem II for effective diffusion and thermodiffusion) on a periodic unit cell. The contributions of diffusion and dispersion will be discussed. The results have been plotted for two different values of the dimensionless Péclet number defined as $P e=\left\langle\mathbf{v}_{\beta}\right\rangle^{\beta_{\ell_{U C}}} / D_{\beta}$, and also for two cases: touching particles and non-touching particles.

\subsubsection{Pure diffusion ( $\mathrm{Pe}=0$ )}

It has been emphasized in the literature that the "solid-solid contact effect" has a great consequence on the effective thermal
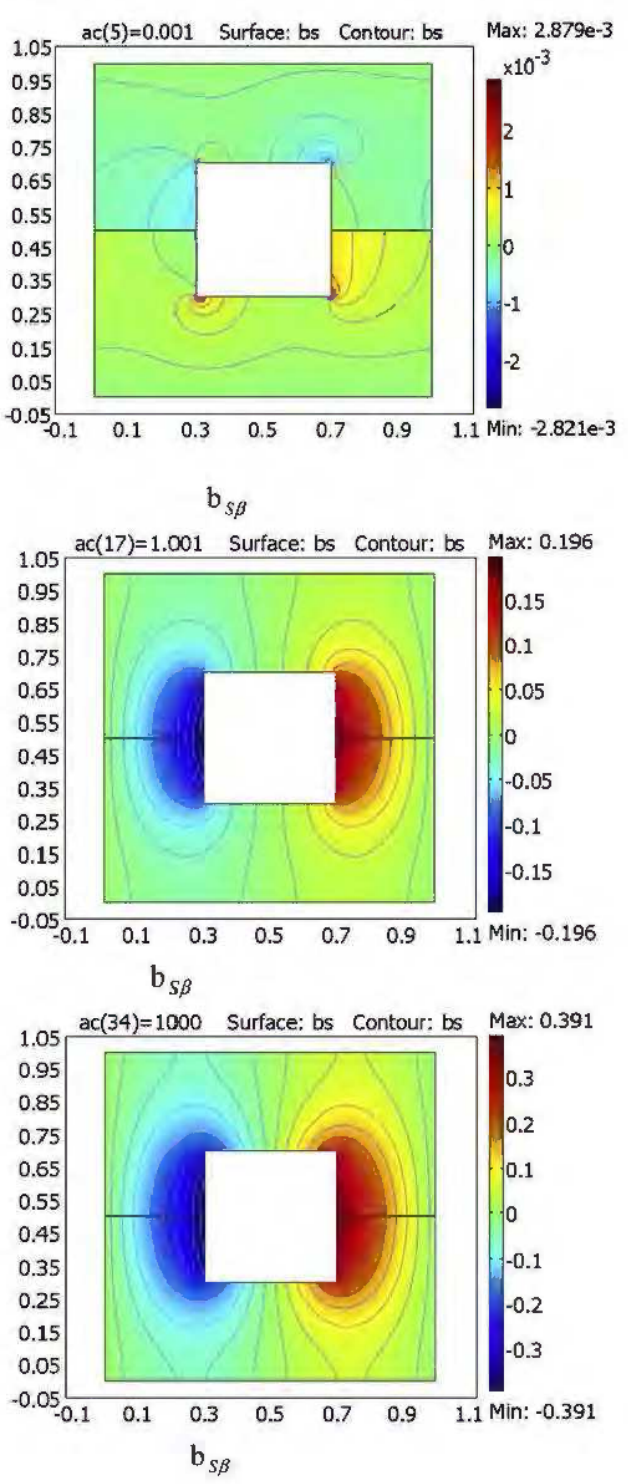

Fig. 6. Comparison of closure variables fields $b_{T \beta}$ and $b_{S \beta}$ when the solid phase is continuous, for different thermal conductivity ratio $(k)$ in the pure diffusion case ( $\alpha / d=0.002$, $\varepsilon_{\beta}=\mathbf{0 . 8}$ ). 
conductivity $[31,37]$. The question is if it can also change the effective thermodiffusion coefficient or not?

In order to model the effect of particle-particle contact we used the model illustrated in Fig. 3, in which the particle-particle contact area is determined by the adjustable parameter a/d (the fraction of particle-particle contact area).

When $a / d=0$, the $\beta$-phase is continuous and the effective conductivity $\left(\mathrm{k}^{*} / \mathrm{k}_{\beta}\right)$ initially increases with an increase in the thermal conductivity ratio $\left(\kappa=k_{\sigma} / k_{\beta}\right)$ and then reaches an asymptote. In addition, it was found that the thermal conductivity ratio has no influence on the thermodiffusion coefficient in the pure diffusive case [9].

When a/d is not zero, at large values of $k$, the solution predicts a linear dependence of $k^{*} / k_{\beta}$ on the ratio $k$. The calculated results, obtained in this study, for both the continuous $\beta$-phase (nontouching particles) and the continuous $\sigma$-phase (touching particles) are shown in Fig. 4.

Here, for the symmetric unit cell shown in Fig. 3, and for the pure diffusive case, one has $k^{*}=\left.k_{\beta}^{*}\right|_{x x}=\left.k_{\beta}^{*}\right|_{y y}$.

As we mentioned before, the selection of $a / d=0.002$ gives the closest agreement with the available experiment data [37]. At this point, it must be emphasized that the area of contact has a strong impact on the results. This area is very sensitive to the grain geometry, the material properties, the mechanical state, etc. This suggest that the value $a / d=0.002$ is not general and may depend on the above mentioned factors.

In this study, the particle-particle contact fraction ( $a / d)$ of 0.002 has been chosen, in order to compare our results with the ones published in the cited references.

Unfortunately, we cannot use this type of geometry to study the effect of solid-solid connection on the effective thermodiffusion coefficient in a two-dimensional system because the fluid phase is not continuous and fluid advection cannot occur in this case. We have used therefore a different geometry which has only a particle connection in the $x$-direction as shown in Fig. 5 . This unit cell is not symmetric and therefore, for an arbitrary effective tensor $\Phi^{*}$, we have in principale $\left.\varphi_{\beta}^{*}\right|_{x x} \neq\left.\varphi_{\beta}^{*}\right|_{y y}$.

In this paper, because of the general objectives of this study, only the $x x$-component of the effective tensors $\left(\left.\varphi_{\beta}^{*}\right|_{x x}\right)$ have been calculated.

The a/d ratio has been selected to be 0.002 as mentioned before. The closure problems have been solved on the geometry shown in Fig. 5 in a purely diffusive case. Comparisons of fields $\mathbf{b}_{T \beta}$ and $\mathbf{b}_{5 \beta}$ when the solid phase is continuous, for different thermal conductivity ratios $(\kappa)$ and a pure diffusive case are shown in Fig. 6. As we can see, the closure variable for concentration $b_{S \beta}$ also changes with the conductivity ratio.

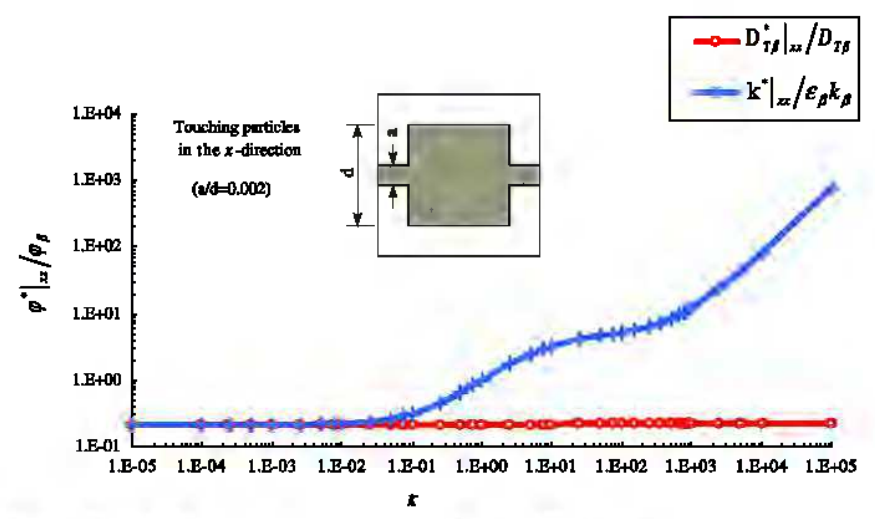

Fig. 7. Effective thermal conductivity and thermodiffusion coefficient for touching particles, $a / d=0.002, \varepsilon_{\beta}=0.36$ and at pure diffusion.

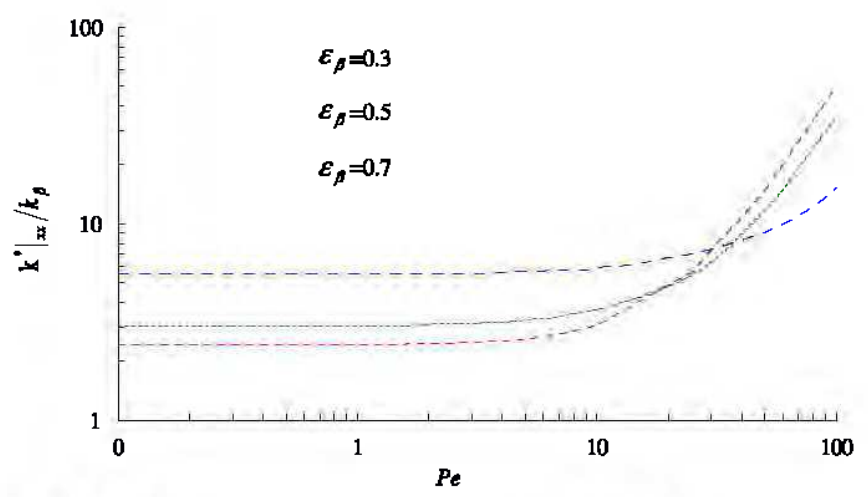

Fig 8. Effective longitudinal thermal conductivity coefficients for non-touching $(a / d=0)$ particles $\left(\varepsilon_{\beta}=0.36\right)$, in the convective case $P e=2$ and different porosities.

The $x x$-components of the effective thermal conductivity and thermodiffusion coefficients, using the geometry shown in Fig. 5, are plotted in Fig. 7. Despite the results illustrated in Fig. 4, the ratio $\left.k^{*}\right|_{x x} / k_{\beta}$ becomes constant for small values of $x$ because in the $y$ direction there is no particle-particle resistance. At large values of $\kappa$, the solution predicts a linear dependence of $\left.k^{*}\right|_{x x} / k_{\beta}$ on the ratio $\kappa$. However, the ratio $\left.D_{T \beta}^{*}\right|_{\gamma \alpha} / D_{T \beta}$ remains constant and equal to 0.59 whatever the thermal conductivity ratio $k$.

Here, for a non-conductive solid phase (or very poor solid conductivity), one can write (See also reference [9])

$\frac{\left.D_{\beta}^{*}\right|_{x x}}{D_{\beta}}=\frac{\left.D_{T \beta}^{*}\right|_{x x}}{D_{T \beta}}=\frac{\left.k^{*}\right|_{\chi x}}{\varepsilon_{\beta} k_{\beta}}=\frac{1}{\tau}=0.59$

where $\tau$ is the scalar tortuosity of the porous matrix.
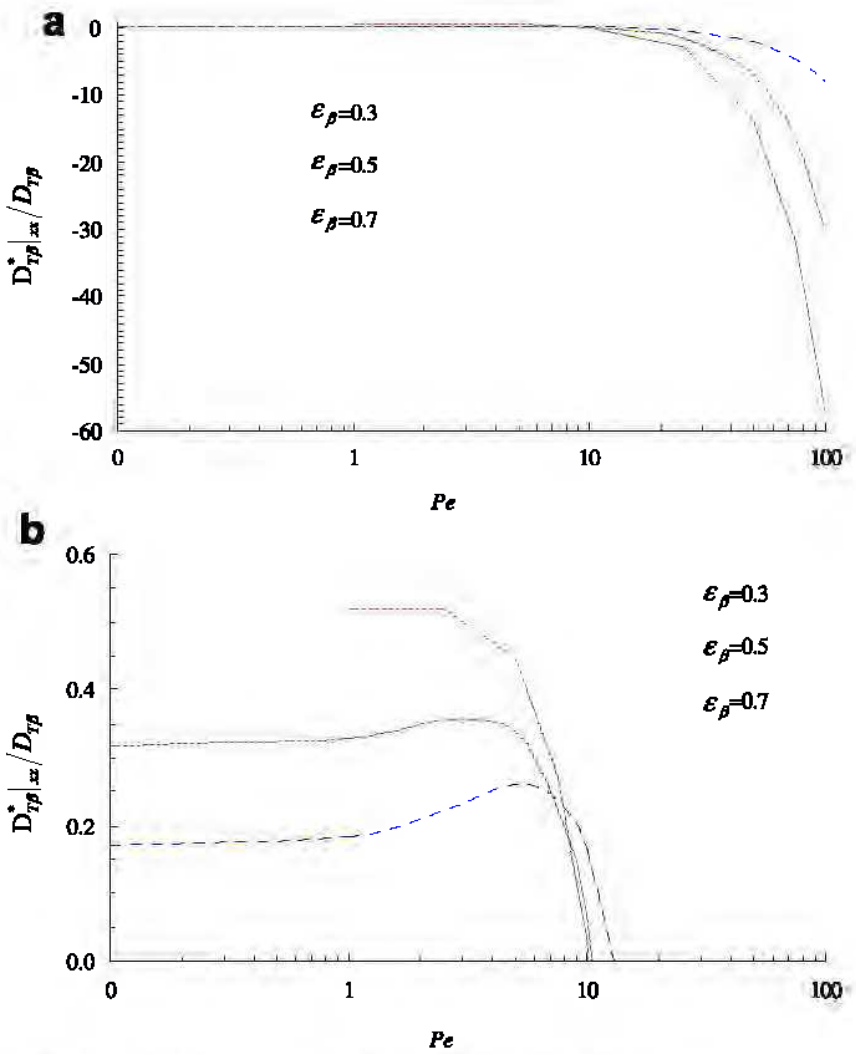

Fig 9. (a) Effective longitudinal thermodiffusion coefficients for non-touching $(a / d=0)$ particles $\left(e_{\beta}=0,36\right)$, in the convective case $P \varepsilon=2$ and different porosities, (b) the positive values. 


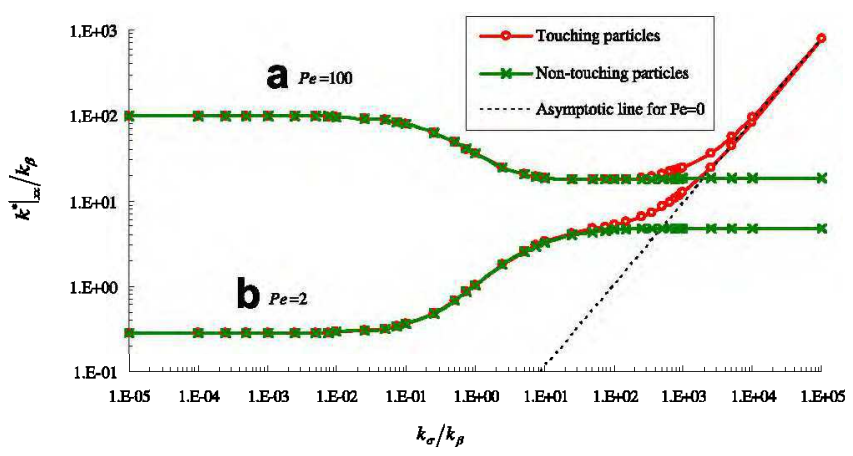

Fig. 10. Effective longitudinal thermal conductivity for non-touching $(a / d=0)$ and touching ( $a / d=0.002$ ) particles $\left(\varepsilon_{\beta}=0.36\right.$ ), in the convective cases: (a) $P e=2$, (b) $\mathrm{Pe}=100$.

\subsubsection{Convective regime ( $\mathrm{Pe}>1$ )}

We have seen in the previous section that the thermal conductivity ratio has no impact on the ratio $\left.D_{T \beta}^{*}\right|_{x x} / D_{T \beta}$. This is coherent with our previous fundings in Davarzani et al. (2010). We already showed in this paper that this conclusion does not hold for the dispersive regime. In this section, we review this question in the case of touching particles.

In order to study this influence of the solid contact on the effective coefficients in the convective regime, the closure problems have been solved using the geometry shown in Fig. 5, with an imposed horizontal pressure gradient. Two series of calculation are made for touching particles $(a / d=0.002)$ and non-touching particles $(a / d=0)$.

During the numerical simulation for different cases, we remarked that the impact of dispersion on the effective thermodiffusion coefficients depends also on the porosity. Here, we have first studied the influence of the porosity on the effective coefficients, for non-touching cases. The effective longitudinal thermal conductivity coefficients versus the Péclet number and for different $\beta$-phase porosity ( $\varepsilon_{\beta}=0.3,0.5$ and 0.7 ) are plotted in Fig. 8. As we can see, the effective thermal conductivity coefficients first decrease when increasing the porosity and then for the large values of the Péclet number the behaviour is inversed.

The evolution of the effective thermodiffusion coefficients for the same porosities versus the Péclet number is shown in Fig. 9(a) for all values and Fig. 9(b) for the positive values. Here, contrary to the effective thermal conductivity, the effective thermodiffusion coefficients first increase when increasing the porosity and then decrease. We can see from Fig. 9(b) very different behaviours for the thermodiffusion coefficients in porous media for small volume fractions of the fluid phase. When the porosity is high, the effective thermodiffusion coefficient is first constant and then decreases when increasing the Pêclet number. However, for a small fluid phase porosity, one can observe a maximum. The effective thermodiffusion coefficient is constant in the diffusive regime, and then increases when increasing the Péclet number up to a maximum value and then decreases when in the convective regime.

The results for the effective thermal conductivity and thermodiffusion coefficients are shown respectively in Figs. 10 and 11. The results have been plotted for two different Péclet numbers $(P e-2$, $P e=100)$, and the dispersion curves have been plotted in Figs. 12 and 13 , for different thermal conductivity ratios. In this study, we have calculated only the longitudinal dispersion coefficients, which are obviously more realistic than the transversal dispersion coefficients, given the lack of connectivity in the $y$-direction for this 2D unit cell.

\section{- Effective thermal conductivity}

As shown in Fig. 10, the longitudinal thermal conductivity, $\left.k^{*}\right|_{\chi x}$ ( $x x$-component of the effective thermal conductivity tensor $\mathbf{k}^{*}$ ), is changed significantly by the contact effect, for conductivity ratios above $\sim 100$.

From Fig. 10 we can see also that the effect behaviour for small Péclet number $(\mathrm{Pe}=2)$ is similar to the pure diffusive case: The
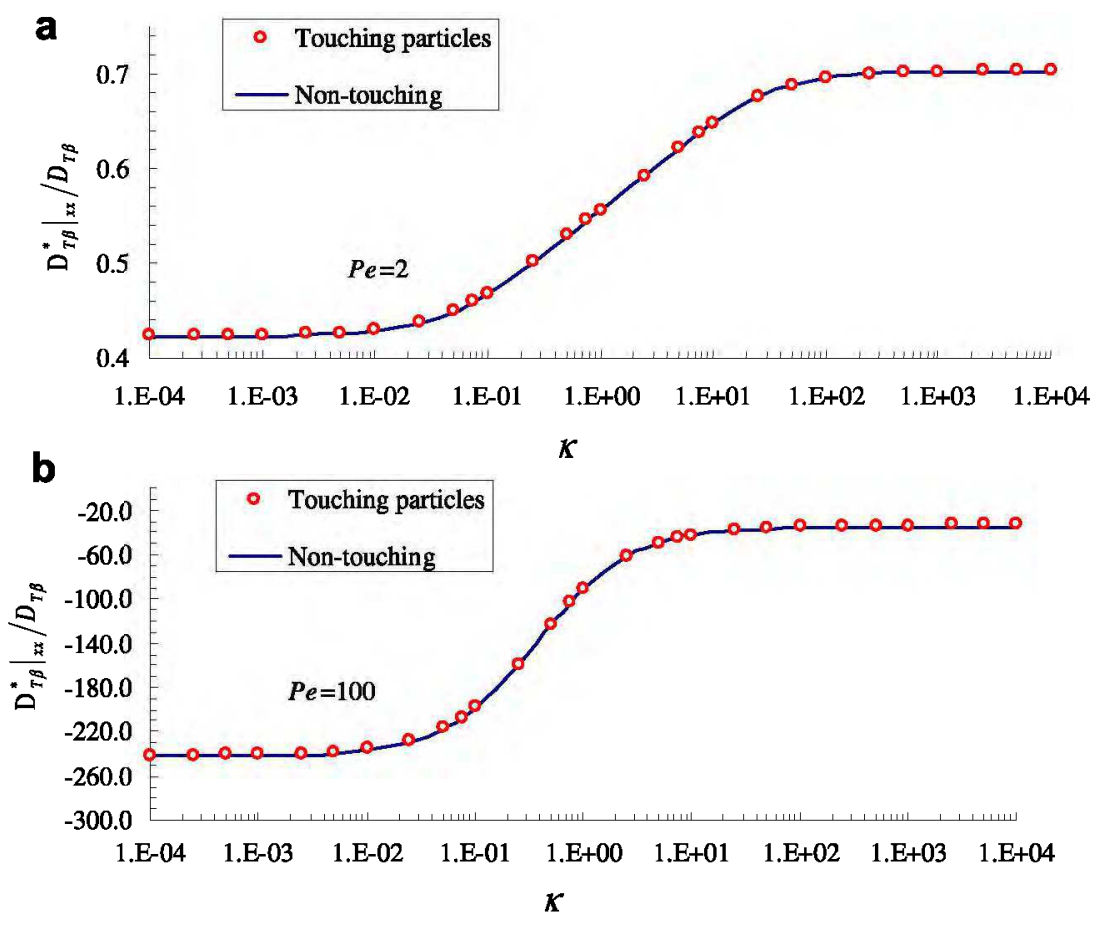

Fig. 11. Effective longitudinal thermodiffusion for non-touching $(a / d=0)$ and touching $(a / d=0.002)$ particles $\left(\varepsilon_{\beta}=0.36\right)$, in the convective cases: (a) $P e=2$, (b) $P e=100$. 
effective conductivity initially increases with an increasing in $\kappa$ for two cases (with and without contact effect). At large values of $\kappa$ the touching particle solution predicts a linear dependence of $\left.k^{*}\right|_{x x} / k_{\beta}$, with $\kappa$ whereas, for the non-touching case, the ratio $\left.k^{*}\right|_{x x} / k_{\beta}$ reaches an asymptote.

For higher Péclet number $(P e=100),\left.k^{*}\right|_{x x} / k_{\beta}$ is decreased by increasing $k$ (with or without contact effect). After a transition, the effective coefficient increases linearly with $\kappa$ for the touching particle case and becomes constant for non-touching particles. This figure also reveals that the starting point of the linearity relation at large values of the conductivity ratio increases when increasing the $P e$ number. We see that the difference between the values of $\left.k^{*}\right|_{x x} / k_{\beta}$ decreases when increasing $k$. However, there is a striking difference between the touching particles and non-touching particles cases. For non-touching particles, there is an additional thermal dispersivity due to the dispersion effect.

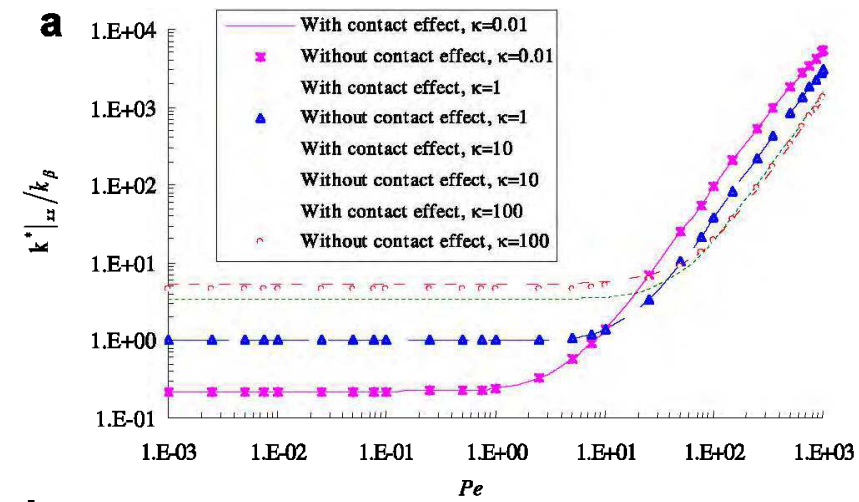

b
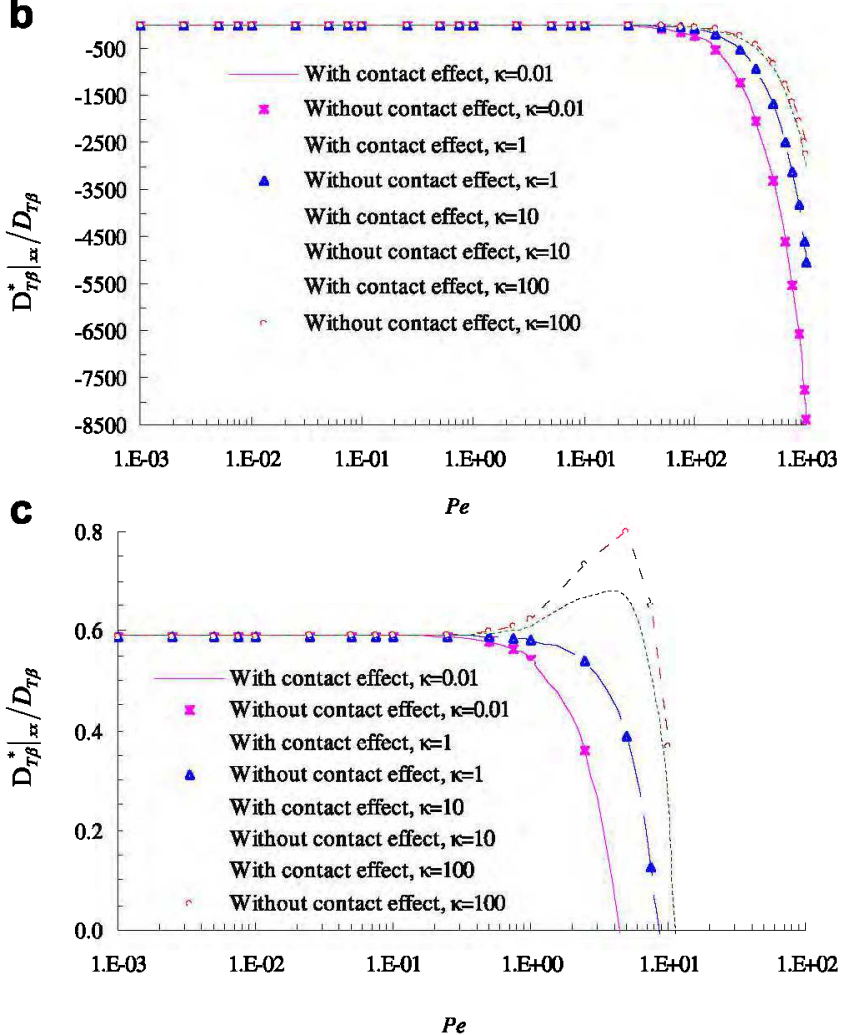

Fig. 12. Effective longitudinal (a) thermal conductivity, (b) thermodiffusion, and (c) the positive values of the effective thermodiffusion coefficients for non-touching ( $a / d=0$ ) and touching ( $a / d=0.002$ ) particles $\left(\varepsilon_{\beta}=0.36\right.$ ) for different thermal conductivity ratios $(k<100)$
Therefore, the asymptote for $P e=100$ is higher than for $P e=2$. In the case of touching particles, the increase in effective conductivity due to higher and higher values of $\kappa$ becomes larger and larger than the "constant" additional value due to dispersion. Therefore, dispersion becomes negligible and all values for all $\mathrm{Pe}$ numbers would merge into the linear relationship for $P e=0$.

\section{- Effective diffusion and thermodiffusion}

The effective longitudinal thermodiffusion coefficients for different Péclet numbers ( 2 and 100 ), with and without contact effects are plotted in Fig. 11. This figure shows clearly that the contact effect does not change the effective thermodiffusion coefficient for all values of thermal conductivity ratio.

The effective longitudinal dispersion ratio $\left.D_{\beta}^{*}\right|_{x x} / D_{\beta}$ depends on the porosity, Péclet number and geometry. This ratio is about 0.78
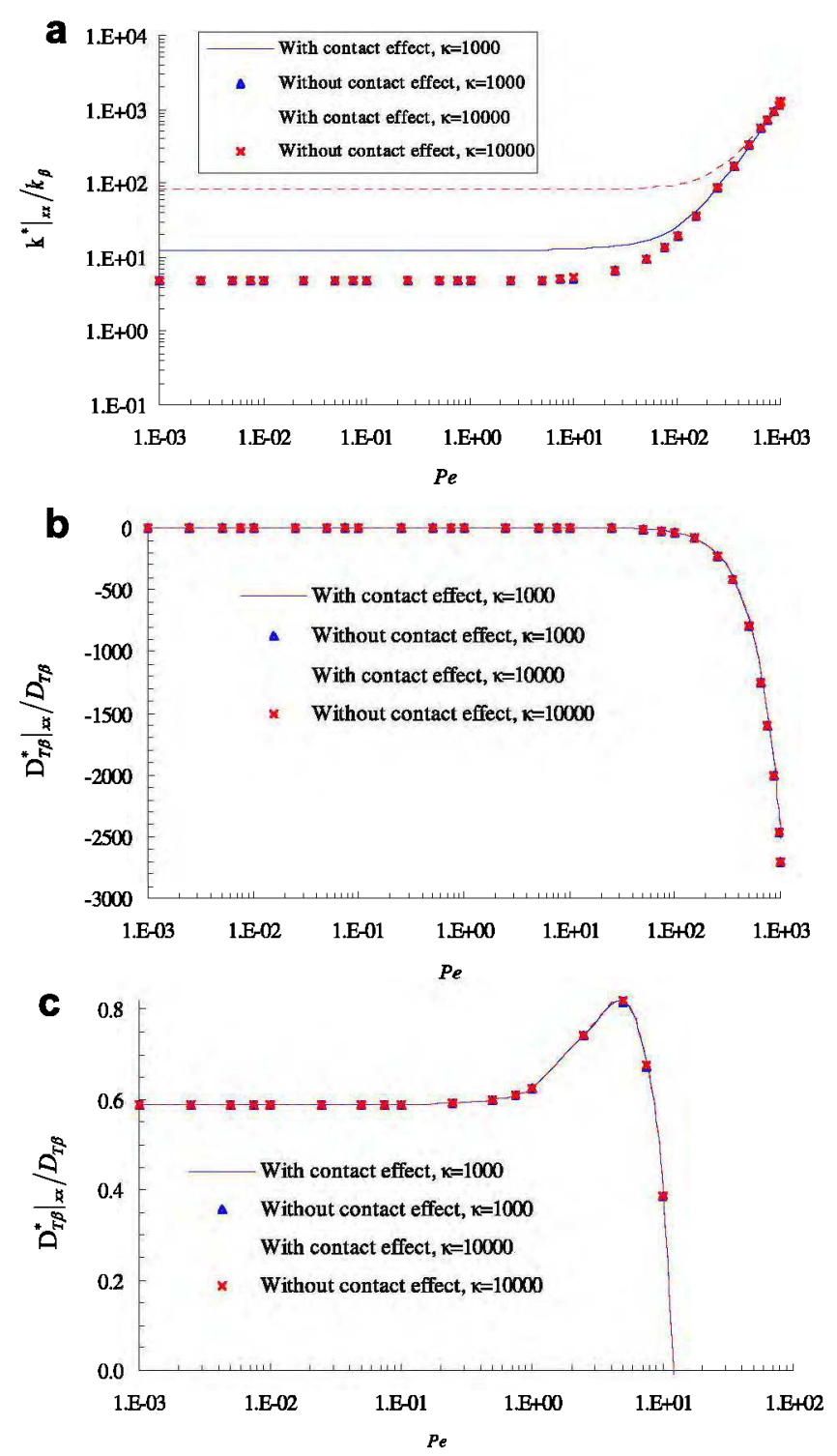

Fig. 13. Effective longitudinal (a) thermal conductivity, (b) thermodiffusion, and (c) the positive values of the effective thermodiffusion coefficients for non-touching ( $a / d=0$ ) and touching ( $a / d=0.002$ ) particles $\left(\varepsilon_{\beta}=0.36\right.$ ) for different thermal conductivity ratios $(\kappa>100)$ 


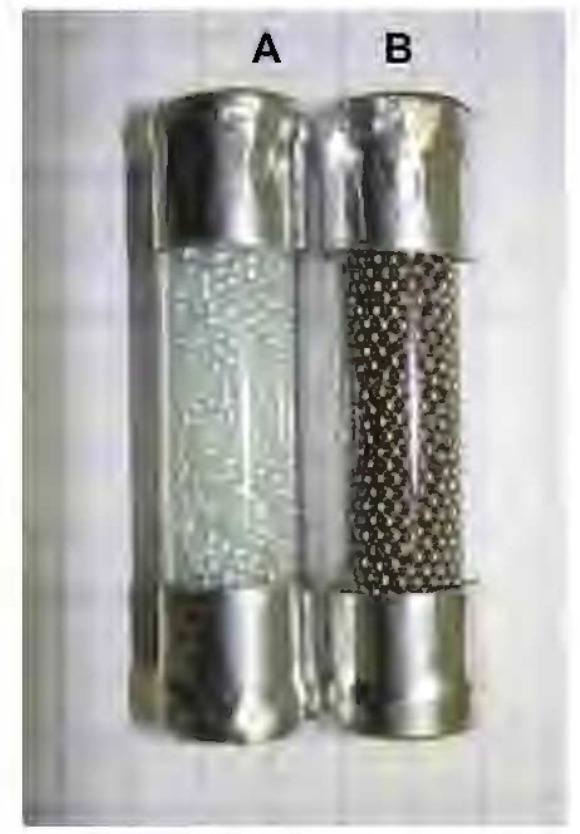

Fig. 14. Cylindrical samples filled with different materials (A: glass spheres, B: stainless steal and $\varepsilon=42.5$ ).

for $P e=2$, with and without contact effect. This ratio for $P e=100$ is about 270.9, without contact effect and becomes about 269.5, with solid contact effect. This small difference comes from the difference of the geometry between two models.

The curves of dispersion for different thermal conductivity ratio have been plotted in Figs. 12 and 13.

As shown in Fig. 12a for thermal conductivity and Fig. 12b for thermodiffusion, the influence of the contact effect is not considerable when $k<100$.

For the large value of the $k$, even if the influence of contact effect on the effective thermal conductivity is important (Fig. 13a), the effective thermodiffusion coefficients remain independent (Fig. 13b).

From these results, we can conclude also that the separation rate in a packed thermogravitational cell when dispersion is important must be influenced by the thermal conductivity ratio.

A direct numerical simulation model has been proposed recently by Davarzani and Marcoux (2011) to study the influence of porous matrix thermal properties on the separation rate in a model of packed thermogravitational column saturated by a binary mixture [7]. The results show that the separation rate is changed significantly by the conductivity ratio of the solid/fluid phases [7]. This may be one of the reasons of discrepancy which exists between theoretical and experiment results in a packed thermogravitational cell (see also reference [11]).

In the next section, in order to validate the theoretical results obtained in this study, experiences have been done using two-bulb thermodiffusion cell in the purely diffusive case.

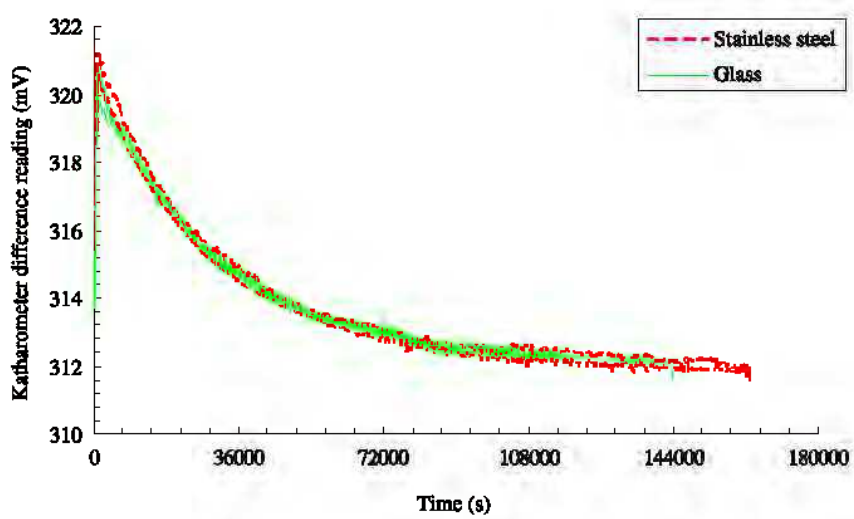

Fig. 15. The kinetic of Katharometer reading in thermodiffusion cell for $\mathrm{He}-\mathrm{CO}_{2}$ binary mixture and for different materials ( 3 samples of stainless steal and 3 samples of glass spheres) $(\Delta T=50 \mathrm{~K})$

\subsection{Experimental results}

The experiences have been done to study the influence of thermal conductivity ratio and solid contact effect on the effective thermodiffusion coefficients.

\subsubsection{Effect of solid thermal conductivity on thermodiffusion}

In Section 5.1, the theoretical model revealed that, for pure diffusion, the effective thermodiffusion coefficients are independent of the thermal conductivity ratio and particle-particle contact. To validate this result we have conducted some experiments with two different materials as shown in Fig. 14: Stainless steel and glass spheres.

The material physical properties are listed in Table 1.

The inner diameter of the insulated, rigid, diffusion glass tube is chosen to be $0.75 \mathrm{~cm}$ and the length is $4 \mathrm{~cm}$. The gas mixture used for this study is a $\mathrm{He}(50 \%)-\mathrm{CO}_{2}(50 \%)$ mixture. The measurement protocol goes as follows. The tubes filled by spheres have a porosity of about $\varepsilon=42.5$. First, the sample is placed carefully between the two bulbs. Air is extracted from the system using a vacuum pump. Then, the system, which is kept at a uniform temperature of $325 \mathrm{~K}$, is filled by the gas mixture at atmospheric pressure. To start the thermodiffusion process, the temperature of the top bulb is increased to $T_{H}=350 \mathrm{~K}$ and, at the same time, the temperature of the bottom bulb is decreased to $T_{C}=300 \mathrm{~K}$. The advantage of this method is that, at the end of this step, when the temperature of each bulb is constant, the pressure of the closed system will be the same as at the starting of the experiment. During this intermediate period, the thermodiffusion process is negligible because of the forced convection between the two bulbs through the tube. In such a system heated at the top and cooled at the bottom, natural convection is negligible. Then, continuous measurements by katharometer, barometers, and thermometers are started in the two bulbs.

Since there are some slight variations in the porous medium between two different experiments (because of packing degree, even if the grain sphere diameter does not change), three samples of each material were used for the measurements.

Table 1

Solid (spheres) and fluid mixture physical properties $(T=300 \mathrm{~K})$ - configuration 1 [22]

\begin{tabular}{|c|c|c|c|c|c|c|c|}
\hline Material of particles & $\begin{array}{l}\text { Diameter } \\
(\mathrm{mm})\end{array}$ & $\begin{array}{l}k_{\sigma} \text { (sphere) } \\
(\mathrm{W} / \mathrm{m} \mathrm{K})\end{array}$ & $\begin{array}{l}k_{\beta} \text { (gases) } \\
(W / \mathrm{m} \mathrm{K})\end{array}$ & $\begin{array}{l}\left(\rho c_{p}\right)_{\sigma} \\
\left(\mathrm{kg} / \mathrm{m}^{3} \times \mathrm{J} / \mathrm{kg} \mathrm{K}\right)\end{array}$ & $\begin{array}{l}\left(\rho c_{p}\right)_{\beta} \\
\left(\mathrm{kg} / \mathrm{m}^{3} \times \mathrm{J} / \mathrm{kg} \mathrm{K}\right)\end{array}$ & $\frac{k_{F}}{k_{\beta}}$ & $\frac{\left(\rho c_{p}\right)_{\sigma}}{\left(\rho c_{p}\right)_{\beta}}$ \\
\hline Stainless steel & 1 & 15 & $\mathrm{He}=0.149$ & $7900 \times 477$ & $\mathrm{He}=0.1624 \times 5200$ & 301 & 3202 \\
\hline Glass & 1 & 1.1 & $\begin{array}{l}\mathrm{CO}_{2}=0.0181 \\
\mathrm{Mix}=0.0499\end{array}$ & $2500 \times 750$ & $\begin{array}{l}\mathrm{CO}_{2}=1.788 \times 844 \\
\mathrm{Mix}=1177\end{array}$ & 22 & 1593 \\
\hline
\end{tabular}




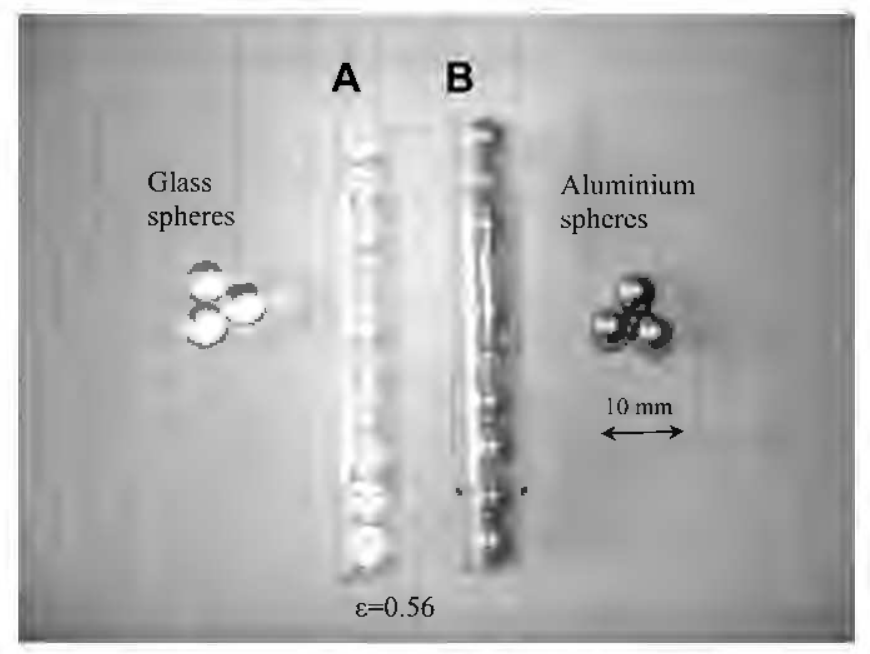

Fig. 16. Cylindrical samples filled with different materials (A: glass spheres, B: aluminium spheres and $\varepsilon=0.56$ )

Katharometer reading time histories for porous media made of spheres with different thermal conductivity (stainless steal and glass spheres) are plotted in Fig. 15.

As it is shown in this plot, thermodiffusion curves for the two different materials can be superimposed and we can conclude that, in this case, the thermal conductivity ratio has no significant influence on the thermodiffusion process. From the formulation discussed in [8], we obtained the ratio of $\left.D_{T \beta}^{*}\right|_{x x} / D_{T \beta}$ equal to about 0.60 .

\subsubsection{Effect of particle-particle touching on thermodiffusion}

The experimental and theoretical results show that the influence of the particle-particle touching on effective thermal conductivity coefficients is considerable when the thermal conductivity ratio is more than 100 as shown also in Fig. 4. Then, to determine the effect of solid phase connectivity, one must use a higher thermal conductor than stainless steal. Therefore, it is better to test more conductive material. We have chosen aluminium and glass spheres with a diameter of $6 \mathrm{~mm}$ shown in Fig. 16. The sample preparation is different from the last section. Here, the sample is made of one column of spheres, in which we can neglect the problem that we had concerning spheres arrangement in the tube to produce exactly the same porosity. Here, inner diameter of insulated rigid glass tube is chosen to be $d=0.75 \mathrm{~cm}$ and length of $\ell=5.8 \mathrm{~cm}$. The number of spheres forming the porous medium is ten, which produced a void fraction about $\varepsilon=0.56$.

The physical properties of two materials used in this experiment are listed in Table 2. As we can see, the thermal conductivity ratio for aluminium and the mixture of helium and carbon dioxide is about 4749 . At this value, the connectivity of the solid phase has a high influence on the effective thermal conductivity coefficients as shown in the theoretical results of Fig. 4.

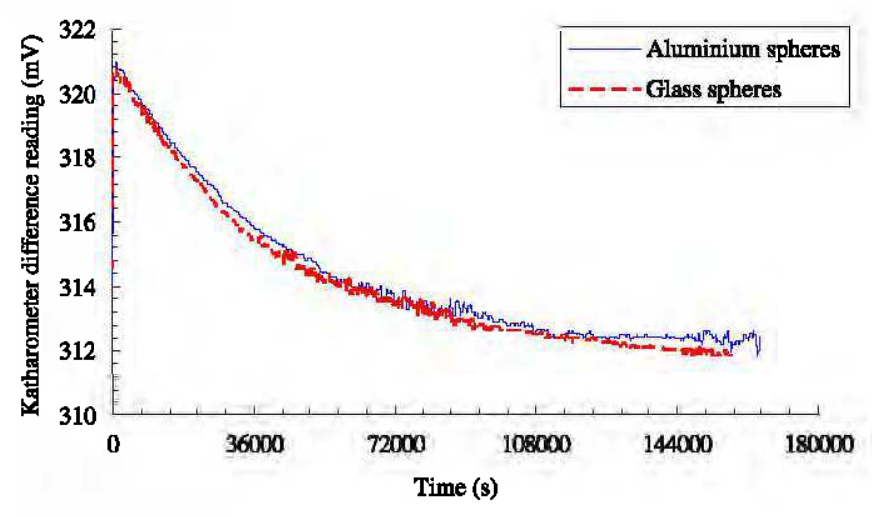

Fig 17. Katharometer time history in two-bulb thermodiffusion cell for $\mathrm{He}-\mathrm{CO}_{2}$ binary mixture (volume fraction of 0.5 ) for porous media made of different thermal conductivity (aluminium and glass spheres) $(\Delta T=50 \mathrm{~K})$

The Katharometer readings for porous media made of aluminium and glass spheres have been plotted in Fig. 17.

This figure shows that the thermodiffusion curves for the two different materials are superimposed. That means that, the particle-particle contact has no influence on the thermodiffusion process, in the pure diffusive case.

It is important to note that these results are just for a pure diffusive system. It must be rementioned that the thermal conductivity ratio has a strong impact for the convective regime.

\section{Conclusion}

In this study, we used a theoretical volume averaging technique and an experimental two-bulb apparatus in order to study the influence of thermal properties of a non-consolidated porous medium on thermodiffusion process taking into account the solid connectivity. The theoretical results show that neither solid thermal conductivity nor particle-particle touching has influence on thermodiffusion for a pure diffusive regime. Contrary, at convective regime the impact of thermal conductivity ratio on the both effective thermal conductivity and thermodiffusion is considerable. In this regime, particle-particle contact has a great influence on the effective thermal conductivity coefficients for the large thermal conductivity contrast of the phases. However, the effective thermodiffusion coefficients do not show significant change with particle-particle touching. We found also that the dispersion effects can be negligible for the large values of the thermal conductivity ratios. For non-consolidated porous media made of spheres with different thermal conductivity coefficients, the experimental results agree with the model obtained by the upscaling technique for effective thermodiffusion coefficients in purely diffusive case.

The authors believe also that in thermogravitational columns, where convection is dominant, the solid thermal conductivity and contact effect will change the separation processes. This may be a very important starting point to conduct new theoretical and experimental studies in the future. In future works, one can also

Table 2

The solid (spheres) and fluid mixture physical properties $(T=300 \mathrm{~K})$ - configuration 2 [22]

\begin{tabular}{|c|c|c|c|c|c|c|c|}
\hline Material of particles & $\begin{array}{l}\text { Diameter } \\
(\mathrm{mm})\end{array}$ & $\begin{array}{l}k_{\sigma} \text { (sphere) } \\
(\mathrm{W} / \mathrm{m} \mathrm{K})\end{array}$ & $\begin{array}{l}k_{\beta} \text { (gases) } \\
(\mathrm{W} / \mathrm{m} \mathrm{K})\end{array}$ & $\begin{array}{l}\left(\rho c_{p}\right)_{\sigma} \\
\left(\mathrm{kg} / \mathrm{m}^{3} \times \mathrm{J} / \mathrm{kg} \mathrm{K}\right)\end{array}$ & $\begin{array}{l}\left(\rho c_{p}\right)_{\beta} \\
\left(\mathrm{kg} / \mathrm{m}^{3} \times \mathrm{J} / \mathrm{kg} \mathrm{K}\right)\end{array}$ & $\frac{k_{\sigma}}{k_{\beta}}$ & $\frac{\left(\rho C_{p}\right)_{\sigma}}{\left(\rho C_{p}\right)_{\beta}}$ \\
\hline $\begin{array}{l}\text { Aluminium } \\
\text { Glass }\end{array}$ & $\begin{array}{l}6 \\
6\end{array}$ & $\begin{array}{r}237 \\
1.1\end{array}$ & $\begin{array}{l}\mathrm{He}=0.149 \\
\mathrm{CO}_{2}=0.0181 \\
\mathrm{Mix}=0.0499\end{array}$ & $\begin{array}{l}2702 \times 903 \\
2500 \times 750\end{array}$ & $\begin{array}{l}\mathrm{He}=0.1624 \times 5200 \\
\mathrm{CO}_{2}=1.788 \times 844 \\
\mathrm{Mix}=1177\end{array}$ & $\begin{array}{r}4749 \\
22\end{array}$ & $\begin{array}{l}2073 \\
1593\end{array}$ \\
\hline
\end{tabular}


resolve the closure problem using a three dimensional model, keeping the $x, y$ and $z$ touching.

\section{References}

[1] A. Bahloul, M.A. Yahiaoui, P. Vasseur, L Robillard, Thermogravitational separation in a vertical annular porous layer, International Communications in Heat and Mass Transfer 31 (6) (2004) 783-794.

[2] A. Bejan, D.A. Nield, Convection in Porous Media. Springer Verlag, 1998.

[3] D. Braun, A. Libchaber, Trapping of DNA by thermophoretic depletion and convection, Physical Review Letters 89 (18) (2002) 188103.

[4] R.G. Carbonell, S. Whitaker, Heat and Mass Transfer in Porous Media. Fundamentals of Transport Phenomena in Porous Media. Martinus Nijhoff, Dordrecht, 1984

[5] P. Costeseque, T. Pollak, J.K. Platten, M. Marcoux, Transient-state method for coupled evaluation of Soret and Fick coefficients, and related tortuosity factors, using free and porous packed thermodiffusion cells, European Physical Journal E, Soft Matter 15 (3) (2004) 249-253.

[6] J.H. Cushman, L.S. Bennethum, B.X. Hu, A primer on upscaling tools for porous media, Advances in Water Resources 25 (8-12) (AUG-DEC 2002) 1043-1067.

[7] H. Davarzani, M. Marcoux, Influence of solid phase thermal conductivity on species separation rate in packed thermogravitational columns: a direct numerical simulation model, Comptes Rendus Mecanique 339 (2011) 355-361.

[8] H. Davarzani, M. Marcoux, P. Costeseque, M. Quintard, Experimental measurement of the effective diffusion and thermodiffusion coefficients for binary gas mixture in porous media, Chemical Engineering Science 65 (18) (2010) 5092-5104.

[9] H. Davarzani, M. Marcoux, M. Quintard, Theoretical predictions of the effective thermodiffusion coefficients in porous media, International Journal of Heat and Mass Transfer 53 (2010) 1514-1528.

[10] H.A. Daynes, Gas Analysis by Measurement of Thermal Conductivity. Cambridge University Press, Cambridge, UK, 1933, pp. 1-302.

[11] D. Fargue, Ph. Jamet, P. Costesque, Dispersion phenomena in thermal diffusion and modeling of thermogravitational experiments in porous media, Transport in Porous Media 30 (1998) 323-344.

[12] A. Firoozabadi, Thermodynamics of Hydrocarbon Reservoirs. McGraw-Hill, New York City, 1991

[13] G. Galliero, J. Colombani, P.A. Bopp, B. Duguay, J.P. Caltagirone, F. Montel, Thermal diffusion in micropores by molecular dynamics computer simulations, Physica A: Statistical Mechanics and its Applications 361 (2) (2006) 494-5101.

[14] Ph. Georis, F. Montel, S. Van Vaerenbergh, Y. Decroly, J.C. Legros, Measurement of the Soret coefficient in crude oil, in: Proceedings of the European Petroleum Conference (1998)

[15] K.E. Grew, W.A. Wakeham, A redetermination of the thermal diffusion factor for some inert gas mixtures, Journal of Physics B: Atomic and Molecular Physics 4 (11) (1971) 1548-1563.

[16] H.R. Heath, T.L. Ibbs, N.E. Wild, The diffusion and thermal diffusion of hydrogen-deuterium, with a note on the thermal diffusion of hydrogenhelium, Proceedings of the Royal Society of London. Series A, Mathematical and Physical Sciences 178 (974) (1941) 380-389 9749.

[17] P. Humphreys, A.E. Gray, Thermal diffusion as a probe of binary diffusion coefficients at elevated temperatures. i. Techniques, and results for carbon dioxide + nitrogen, Proceedings of the Royal Society of London. Series A, Mathematical and Physical Sciences 320 (1542) (1970) 397-415 77835.

[18] T.L. Ibbs, S. Chapman, Some experiments on thermal diffusion, Proceedings of the Royal Society of London. Series A, Containing Papers of a Mathematical and Physical Character 99 (700) (1921) 385-397 93924.

[19] I. Ryzhkov Ilya, On double diffusive convection with Soret effect in a vertical layer between co-axial cylinders, Physica D 215 (2) (2006) 191-200.

[20] G. Jessop, Katharometers, Journal of Scientific Instruments 43 (11) (1966) $777-782$

[21] C.G. Jiang, T.J. Jaber, H. Bataller, M.Z. Saghir, Simulation of Ludwig-Soret effect of a water-ethanol mixture in a cavity filled with aluminum oxide powder under high pressure, International Journal of Thermal Sciences 47 (2008) 126-135.
[22] M. Kaviany, Principles of Heat Transfer. John Wiley \& Sons, Inc, New York, 2002

[23] J. Kreft, Y.L. Chen, Thermal diffusion by brownian-motion-induced fluid stress, Physical Review E 76 (2007) 021912.

[24] B. Lacabanne, S. Blancher, R. Creff, F. Montel, Soret effect in multicomponent flow through porous media: local study and upscaling process, Lecture Notes in Physics 584 (2002) 448-465 Springer.

[25] C. Ludwig, Diffusion zwischen ungleich erwärmten Orten gleich zusammengesetzter Lösungen. Sitzungsberichte der Kaiserlichen Akademie der Wissenschaften Wien, Mathematisch Naturwissenschaftliche Classe 20 (1856) 539.

[26] E.A. Mason, M. Islam, S. Weissman, Thermal diffusion and diffusion in hydrogen-krypton mixtures, Physics of Fluids 7 (7) (1964) 1011-1022.

[27] I. Nozad, R.G. Carbonell, S. Whitaker, Heat-conduction in multiphase systems. 1. Theory and experiment for 2-phase systems, Chemical Engineering Science 40 (5) (1985) 843-855.

[28] I. Nozad, R.G. Carbonell, S. Whitaker, Heat-conduction in multiphase systems. 2. Experimental-method and results for 3-phase systems, Chemical Engineering Science 40 (5) (1985) 857-863.

[29] J.K. Platten, The Soret effect: a review of recent experimental results, Journal of Applied Mechanics 73 (1) (2006) 5-15.

[30] J.K. Platten, J.C. Legros, Convection in Liquids. Springer, Berlin, 1984, Chap. 9.

[31] M. Quintard, Diffusion in isotropic and anisotropic porous systems: threedimensional calculations, Transport in Porous Media 11 (2) (1993) 187-199.

[32] M. Quintard, M. Kaviany, S. Whitaker, Two-medium treatment of heat transfer in porous media: numerical results for effective properties, Advances in Water Resources 20 (2-3) (1997) 77-94.

[33] G.D. Rabinovich, RY. Gurevich, G.N. Bobrova, Thermodiffusion Separation of Liquid Mixtures. Nauka i Tekhnika, Minsk, 1971, (in Russian).

[34] G.D. Rabinovich, Separation of Isotopes and Other Mixtures by Thermal Diffusion. Atomizdat, Moscow, 1981.

[35] D. Reith, F. Mueller-Plathe, On the nature of thermal diffusion in binary Lennard-Jones liquids, Journal of Chemical Physics 112 (2000) 2436-2443.

[36] M.Z. Saghir, C.G. Jiang, M. Chacha, Y. Yan, M. Khawaja, S. Pan, Thermodiffusion in porous media. in: D.B. Ingham, I. Pop (Eds.), Transport Phenomena in Porous Media, vol. III. Elsevier, Oxford, 2005, pp. 227-260.

[37] M. Sahraoui, M. Kaviany, Slip and no-slip temperature boundary conditions at interface of porous, plain media: conduction, International Journal of Heat and Mass Transfer 36 (1993) 1019-1033.

[38] S.C. Saxena, S. Mathur, G.P. Gupta, The thermal conductivity data of some binary gas mixtures involving nonpolar polyatomic gases, Defence Science Journal 16 (4) (1966) 99-112.

[39] A.A. Shapiro, E.H. Stenby, Factorization of transport coefficients in macroporous media, Transport in Porous Media 41 (2000) 305-323.

[40] AG. Shashkov, A.F. Zolotukhina, T.N. Abramenko, B.P. Mathur, Thermal diffusion factors for binary gas systems: $\mathrm{Ar}-\mathrm{N}_{2}, \mathrm{Ar}-\mathrm{CO}_{2}, \mathrm{He}-\mathrm{H}_{2}, \mathrm{He}-\mathrm{N}_{2} \mathrm{O}, \mathrm{Kr}-$ $\mathrm{N}_{2} \mathrm{O}$ and $\mathrm{He}-\mathrm{NH}_{3}$, Journal of Physics B: Atomic Molecular and Optical Physics 12 (21) (1979) 3619-3630.

[41] C. Soret, Influence de la temperature sur la distribution des sels dans leurs solutions, Compte-Rendu de l'Academie des Sciences 91 (1880) 289 ParisAcademie des Sciences, Paris.

[42] S. Van Vaerenbergh, J.C. Legros, J.L. Daridon, T. Karapantsios, M. Kostoglou, Z.M. Saghir, Multicomponent transport studies of crude oils and asphaltenes in DSC program, Microgravity Science and Technology 18 (3-4) (2006) 150-154.

[43] A van Itterbeek, 0 . van Paemel, J. van Lierde, Measurements on the thermal diffusion in gas mixtures at low temperatures, Physica 13 (4-5) (1947) 231-239.

[44] S. Whitaker, The Method of Volume Averaging. Kluwer Academic Publishers, Dordrecht, The Netherlands, 1999.

[45] S. Wiegand, Thermal diffusion in liquid mixtures and polymer solutions, Journal of Physics: Condensed Matter 16 (10) (2004) 357-379.

[46] V.L. Zhdanov, O.A. Kolenchits, B.P. Mathur, S.C. Saxena, Gas-phase transport phenomena at low temperatures: thermal diffusion in an $\mathrm{Ar}-\mathrm{Kr}$ system, International Journal of Thermophysics 1 (2) (1980) 147-157. 